\title{
Risk factors adversely impacting post coronary artery bypass grafting longer-term vs. shorter-term clinical outcomes
}

\author{
Brendan M. Carr ${ }^{1}$, Frederick L. Grover ${ }^{2}$, Annie Laurie W. Shroyer ${ }^{3,4}$ \\ 'Department of Emergency Medicine, Mayo Clinic, Rochester, MN 55905, USA. \\ 2Department of Surgery, University of Colorado, School of Medicine, Anschutz Medical Campus, Aurora, CO 80045, USA. \\ ${ }^{3}$ Department of Surgery, Stony Brook University, School of Medicine, Stony Brook, NY 11794, USA. \\ ${ }^{4}$ Departmenf of Surgery, Northport Veterans Affairs Medical Center, Northport, NY 11768, USA.
}

Correspondence to: Dr. Annie Laurie W. Shroyer, Tenured Professor and Vice Chair for Research, Department of Surgery, Stony Brook University School of Medicine, Health Sciences Center Level 19, Room \#083, 100 Nicolls Road, Stony Brook, New York, NY 11794-8191, USA. E-mail: annielaurie.shroyer@stonybrookmedicine.edu

How to cite this article: Carr BM, Grover FL, Shroyer ALW. Risk factors adversely impacting post coronary artery bypass grafting longer-term vs. shorter-term clinical outcomes. Vesse/ P/us 2020;4:12. http://dx.doi.org/10.20517/2574-1209.2020.01

Received: 2 Jan 2020 First Decision: 4 Feb 2020 Revised: 21 Feb 2020 Accepted: 9 Mar 2020 Published: 11 May 2020

Science Editor: Mario F. L. Gaudino Copy Editor: Jing-Wen Zhang Production Editor: Tian Zhang

\begin{abstract}
Aim: Coronary artery bypass grafting (CABG) patients' characteristics and surgical techniques associated with shortterm (ST; < 1 year) mortality are well documented; however, the literature pinpointing factors predictive of longer-term (LT; $\geq 1$ year) death rates are more limited. Thus, the CABG factors associated with ST vs. LT mortality were compared.
\end{abstract}

Methods: Using advanced PubMed search techniques, the factors associated with improved post-CABG mortality were compared for ST vs. LT prediction models; ST vs. LT models' results were compared across three time periods: until 1997, 1998-2007, and 2007-2017.

Results: Of 156 post-CABG mortality risk models ( $n=125$ publications), 133 ST and 23 LT models were evaluated. Important predictors consistently included age, ejection fraction, and renal dysfunction/dialysis. The ST models more commonly identified surgical priority, gender, and prior cardiac surgery; however, the LT models more frequently included diabetes and peripheral arterial disease. Compared to ST mortality, patterns also emerged for cerebrovascular disease and chronic lung disease predicting LT mortality. As modifiable risks, body mass index or another marker of body habitus appeared in 31/133 (23\%) of ST models; smoking or tobacco use was considered in only 4/133 (3\%). No models evaluated compliance with ischemic heart disease guidelines. No time period-related differences were found.

Conclusion: Different risk factors predicted ST vs. LT post-CABG mortality; for LT death, debilitating chronic/complex comorbidities were more often reported. As few models focused on identifying modifiable patient risks or ischemic heart disease guideline compliance, future CABG LT risk modeling should address these knowledge gaps.

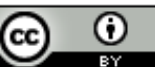

(c) The Author(s) 2020. Open Access This article is licensed under a Creative Commons Attribution 4.0 International License (https://creativecommons.org/licenses/by/4.0/), which permits unrestricted use sharing, adaptation, distribution and reproduction in any medium or format, for any purpose, even commercially, as long as you give appropriate credit to the original author(s) and the source, provide a link to the Creative Commons license, and indicate if changes were made. 
Keywords: Coronary artery bypass graft surgery, risk assessment, outcomes research, survival, mortality

\section{INTRODUCTION}

Over the past 60 years, much has changed in the healthcare field. Increasingly, attention is being paid to healthcare quality with the goals of improving clinical outcomes and increasing value of care delivered. A special emphasis in quality improvement has been placed on high volume procedures such as coronary artery bypass grafting (CABG). Although CABG volumes have declined from 213,700 procedures (2011) to $\sim 156,900$ procedures (2016), it remains the most common cardiac surgical procedure performed in the United States ${ }^{[1-3]}$. To evaluate the true value of CABG, longer-term outcomes are necessary to establish the durability of the procedure. Accordingly, the baseline patient risk factors associated with short-term $(<1$ year $)$ and longer-term ( $\geq 1$ year) CABG mortality were compared.

Interpreting $\mathrm{CABG}$ clinical outcomes data can often be challenging, as there may be a wide range in preCABG patient's severity of coronary disease or comorbidity-related disease complexity, variations in CABG operative techniques used or post-CABG pre-discharge patient care management, as well as providerbased variations for annual CABG volumes performed. In 1972, the Department of Veterans Affairs (VA) healthcare system began internally reporting national unadjusted outcome rates (e.g., "observed" inhospital mortality rates) for patients undergoing cardiac surgery at its institutions; these first VA reports focused upon observed CABG mortality and post-CABG complication rates ${ }^{[4]}$.

After US hospitals' CABG mortality reports were made publicly available by the Department of Health and Human Services in 1985, Congress in 1986 mandated that the VA report risk-adjusted cardiac surgery mortality rates and compare these VA rates to national standards ${ }^{[5]}$. Given these legislation-driven mandates, VA clinicians and scientists began looking for ways to "level the playing field" using statistical risk models to permit more meaningful comparisons between centers and surgeons; these risk-adjusted outcome reports were used in their local VA medical centers' quality improvement endeavors.

Initiated in April 1987, the VA Continuous Improvement in Cardiac Surgery Program (CICSP) was founded; CICSP was one of the first registries to report risk-adjusted CABG 30-day operative mortality and major morbidity across all participating VA hospitals ${ }^{[4]}$. The VA CICSP identified a set of Veteran risk characteristics associated with CABG adverse outcomes; based on gathering 54 patients' risk, cardiac surgical procedural details, and hospital-related outcomes, the VA CICSP calculated the "expected" mortality occurrence for each Veteran undergoing a CABG procedure. Across providers and "highrisk" patient sub-groups, therefore, "observed" to "expected" outcome rates were compared to identify opportunities to improve their local VA cardiac surgical care ${ }^{[6]}$.

Some of the earliest lists of pre-CABG patient risk factors associated with mortality were developed entirely based on expert consensus. As different national, regional, and state-wide databases originally gathered different sets of patient risk factors, an early consensus conference was held to identify the minimal set of "core" risk variables required to be captured ${ }^{[7,8]}$. Given challenges encountered with CABG records' data completeness, however, these earliest mathematical approaches to calculate risk-adjusted outcome rates made use of Bayes theorem ${ }^{[9]}$. Since the VA's programmatic expansions in 1992, dramatic improvements were made in the VA completeness of CABG data captured; thus, logistic regression emerged as the most common analytical approach used. Other approaches have been reported, including applications of neural networks and Cox regression ${ }^{[10,1]}$. Given both the ease of clinical interpretation and superior statistical model performance, however, logistic regression remains the standard analytical approach used to predict post-CABG short-term (ST) and longer-term (LT) mortality ${ }^{[12-14]}$. 
Historically, the process of choosing logistic regression eligible ("candidate") risk variables was different for each $C A B G$ registry. As this pre-selection candidate variable approach may have introduced subjectivity and biased model results, CABG risk models (such as those developed by the VA, Society of Thoracic Surgeons, and EuroSCORE teams) have been derived in recent years using a standardized approach with a core set of model eligible variables. Beyond this core set, however, each database incorporates an expanded set of population-specific risk variables in their risk modeling processes.

Over the past 30 years, nearly countless CABG risk models with various designs and complexity have been developed to predict the likelihood of death at pre-specified time periods. As the standard ST endpoint used, operative mortality was defined as death within 30 days or within the index hospitalization. As operative mortality avoids any potential post-discharge referral bias (e.g., post-CABG hospital discharge to a separate sub-acute care facility), this endpoint was determined to be the most clinically relevant performance metric; it is commonly used to assess the quality of the surgical procedure. Other models have considered LT death during longer periods of follow-up, investigating the durability of the CABG procedure and importance of other risk factors. For ST and LT published risk models, therefore, this study describes the patterns in pre-CABG factors differentially impacting ST vs. LT mortality. Until this report, these patterns had not been previously described. Moreover, this novel report identifies additional opportunities to improve future CABG risk models.

\section{METHODS}

An advanced literature review was undertaken to document published risk factors associated with postCABG mortality. In February 2019, PubMed was searched for all Medline publications using the following terms: "CABG" (Title) OR "coronary artery bypass" (Title) AND "mortality" (Title) OR "risk" (Title) OR "death" (Title) OR "survival" (Title). This yielded 1904 publications. Following a review of all articles for pre-stated inclusion/exclusion criteria, there were a total of 125 included articles with 156 CABG mortality models. Only papers reporting risk models for mortality after an isolated CABG procedure were included; inclusion criteria were otherwise left intentionally broad so as to gather a wide variety of models. Models requiring data from the postoperative period were excluded for the purpose of this review, whereas those employing only preoperative variables [as opposed to preoperative and intraoperative variables (e.g., cardiopulmonary bypass time)] were identified for sub-analysis review. For the 125 publications meeting all inclusion/exclusion criteria, their reference lists were also carefully reviewed for relevant publications to augment the original search strategy's findings.

Working collaboratively under the senior co-authors' guidance, the majority of literature search screening and data extraction were performed primarily by one author (BC). To permit meaningful model comparisons, risks were classified into 91 different common clinical categories. Clinically relevant composite variables were reported based upon database-specific definitions (e.g., "critical preoperative state" and "extracardiac arteriopathy"). Named risk indices (e.g., "Elixhauser Comorbidity Index") were analyzed using their assigned name as a group, rather than being recorded based upon the indices' subcomponents. For the 125 publications evaluated, the set of risk factors identified to be associated with post-CABG ST or LT mortality were compared. Time trends in models' risk factors reported were evaluated across three time periods until 1997, 1998-2007, and 2007-2017.

\section{RESULTS}

One hundred fifty-six post-CABG mortality risk models were identified within 125 different papers. In Appendix A, the full listing of these papers and models can be found in Supplementary Tables 1 and 2.

Of these models, 133 predicted ST CABG mortality. Operative mortality was the most commonly reported ST endpoint, defined as death occurring during the index hospitalization and/or up to 30 days after the 
index surgical procedure. Twenty-three LT CABG mortality models were identified. The longest period of follow-up was seven years, reported by Wu et al. ${ }^{[15]}$ When looking at those models considering only preoperative (i.e., not intraoperative) risk factors, there were 75 ST models and $14 \mathrm{LT}$ models $($ total $=89$ ). As a pre-planned sub-analysis, risk models considering on-pump vs. off-pump CABG and only preoperative risk factors were also compared separately. This identified three ST and one LT models $($ total $=4)$. The complete listing of variables for the ST $v$ s. LT models with frequency counts is included in Table 1.

Overwhelmingly, age was the most common preoperative variable identified to be predictive of ST postCABG mortality, reported in 115 of 125 (86\%) of those models. Of the articles summarized, 22/156 (14.1\%) did not report age as a risk factor. Across these 22 publications, the age-related variability in reporting observed appears to be due in part to their study-specific populations' inherent risk profile. For example, articles focused upon higher risk patient sub-groups (e.g., emergent CABG patients or those experiencing an acute myocardial infarction) commonly did not report age as a post-CABG mortality model finding. Despite this observed pattern, however, there was not a single, simple explanation for the observed inconsistency in age not being reported across all models.

Age was followed by left ventricular ejection fraction (included in 64\% of ST mortality models), surgical case priority or status (59\%), patient gender (57\%), and having undergone a prior cardiac surgical procedure before the index procedure (55\%); these represented the top five most common preoperative variables for predicting ST post-CABG mortality. For LT models, the top five risk factors were age, ejection fraction, diabetes mellitus, peripheral arterial disease, and renal failure. There appeared to be a trend toward cerebrovascular disease and lung disease being more commonly reported by CABG risk models focused upon mortality beyond one year (compared with other variables within that same subset of models), perhaps suggesting debilitating chronic and complex comorbidities are more useful in prediction of LT mortality.

When the results were grouped into early, mid, and late subgroups by year of publication [Tables 2-4], age and ejection fraction remained among the most common risk factors for models throughout those time periods. No definite trends over time were observed in risk factor prevalence for the overall group or the ST or LT model subgroups, although sample size may have impacted the ability to detect such trends, particularly within the subgroups. Results were also similar when considering models that included only preoperative risk factors [Table 5] or those that considered on-pump vs. off-pump CABG [Table 6].

\section{DISCUSSION}

Across the post-CABG follow-up periods, different pre-CABG risk factors predictive of mortality were documented. This literature search revealed dozens of logistic regression models, each reporting different patient risk factors associated with time-varying post-CABG mortality endpoints. As documented by the tables, the ST models found the patient's risk variables related to their severity of coronary disease (e.g., more commonly reported be important predictors), whereas patient's chronic comorbidities (e.g., diabetes, cerebrovascular disease, or pulmonary disease) appeared to be more frequently associated with LT postCABG mortality. Following one-year post-CABG, life expectancy appears to be most strongly impacted by non-cardiac comorbidities than cardiac factors or surgical processes of care. While optimizing CABG patient selection and surgical techniques may be important ST, optimal management of non-cardiac comorbidities may improve post-CABG patients' LT survival. Moreover, across all follow-up time periods, a patient's age, ejection fraction, and renal function (e.g., creatinine or dialysis dependence) were important predictors of post-CABG mortality; these were consistently reported for the ST and LT mortality time periods.

A special sub-analysis was performed for the sub-group of models comprised of preoperative risk factors along with a variable indicating the on-pump vs. off-pump surgical technique. Although there were 


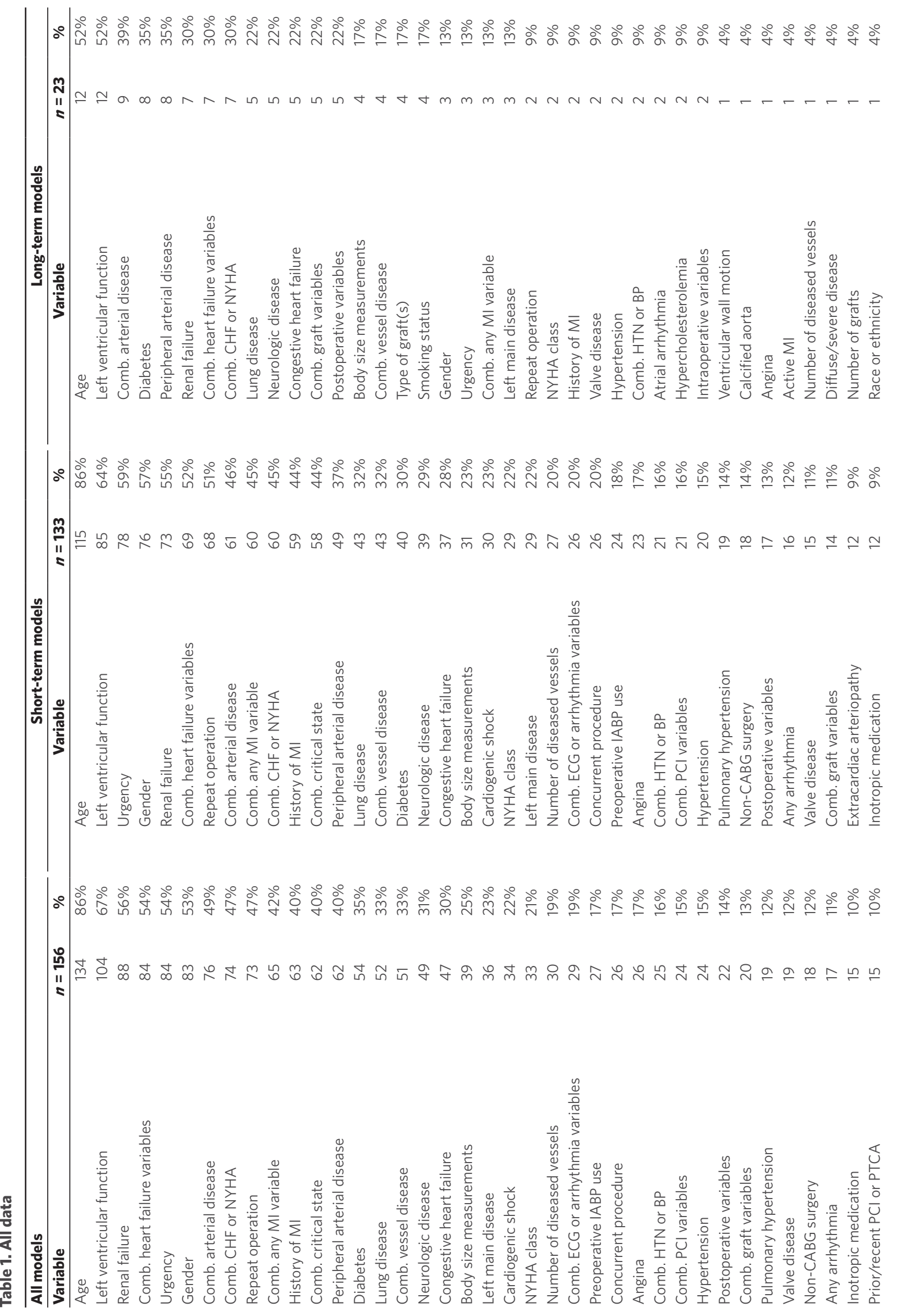




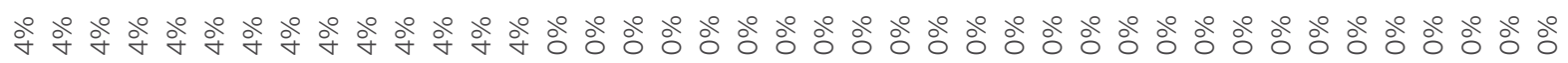

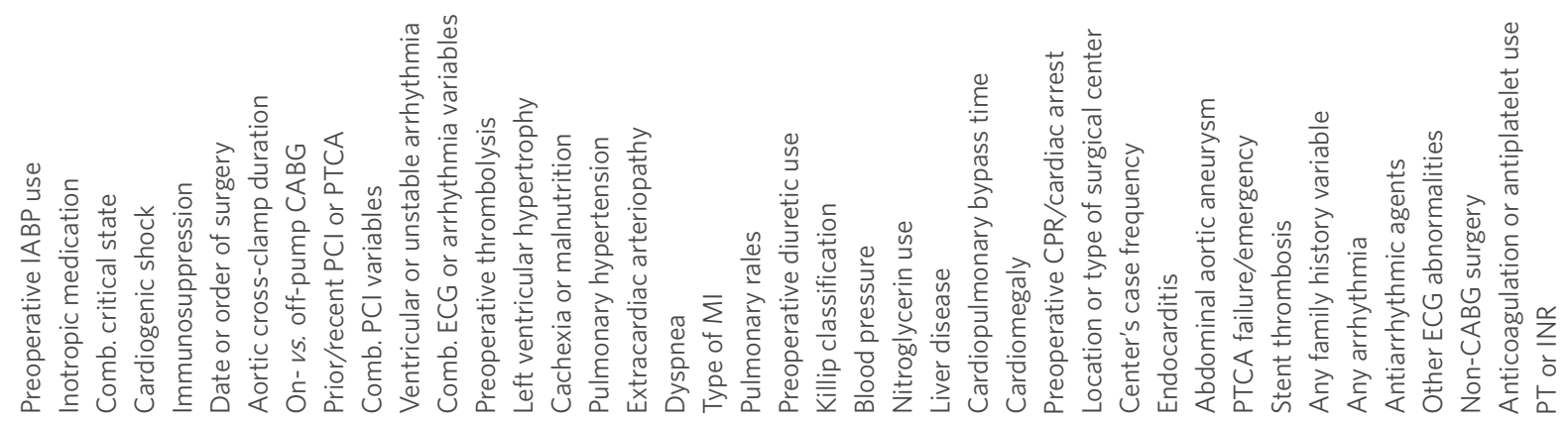

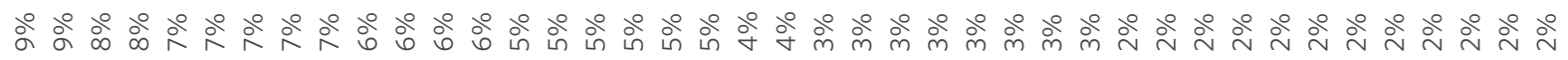

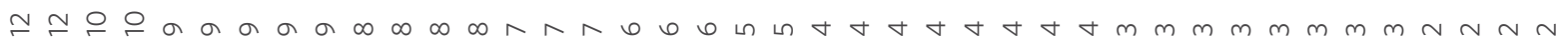

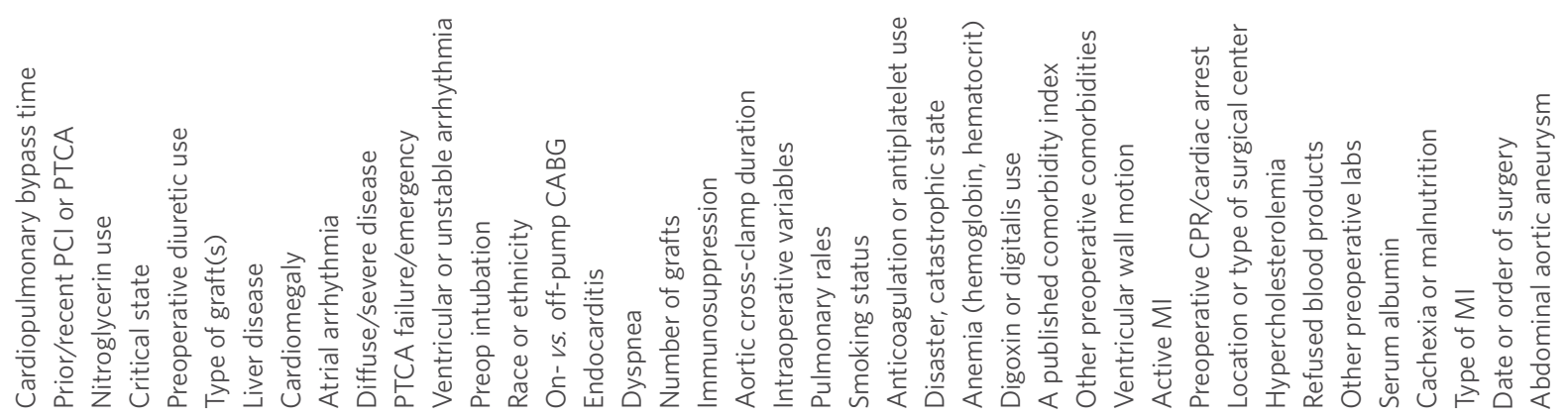

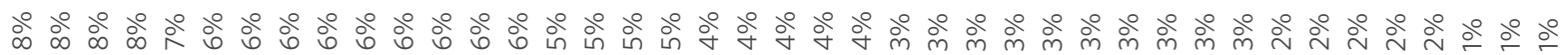

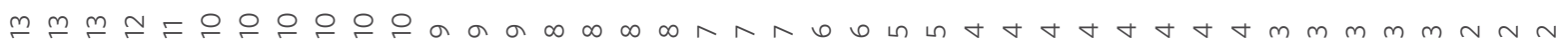

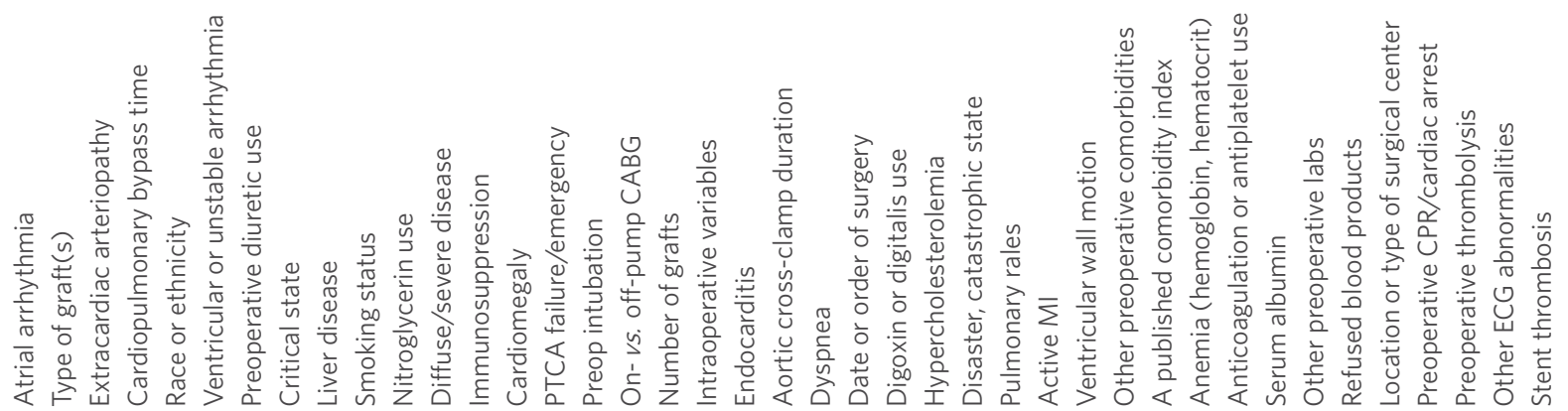




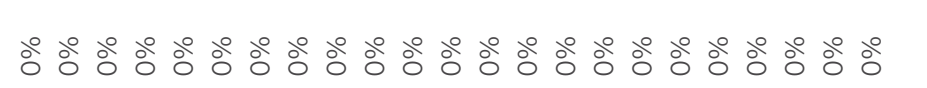

$0000000000000000000000000000 \frac{4}{4}$

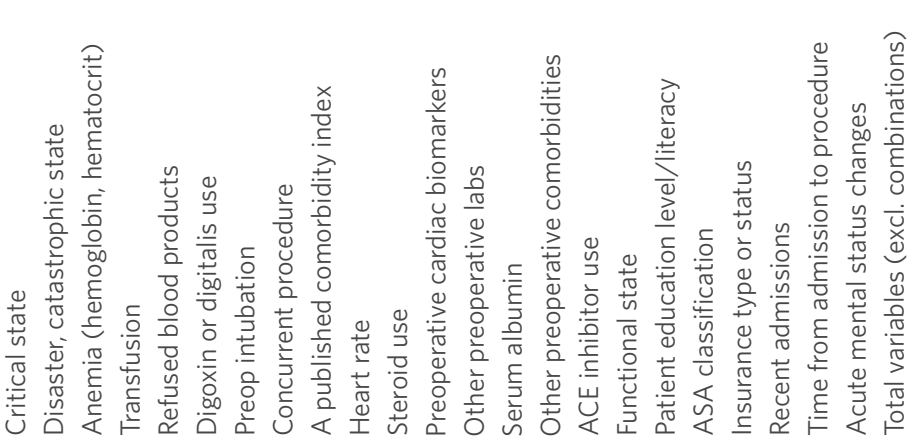

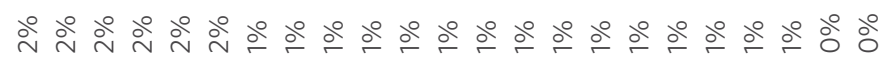

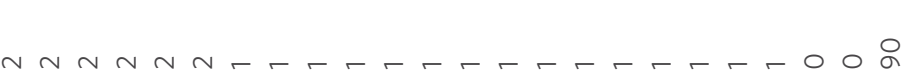

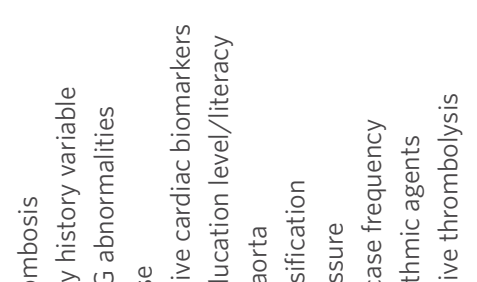

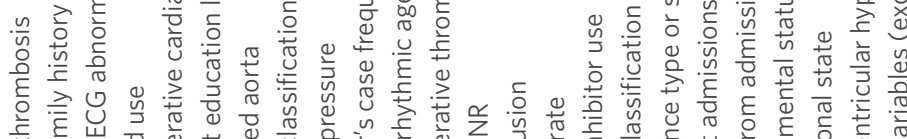

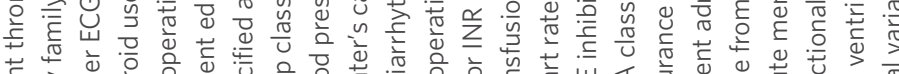

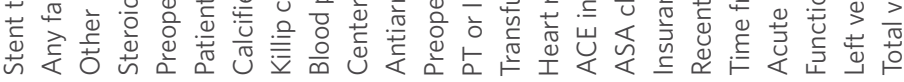

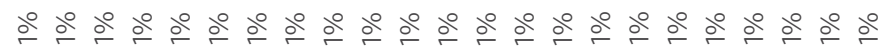

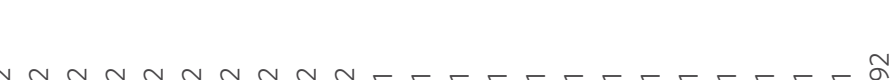

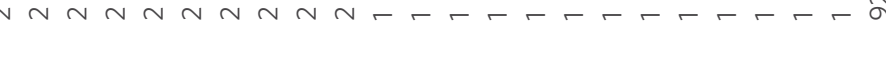

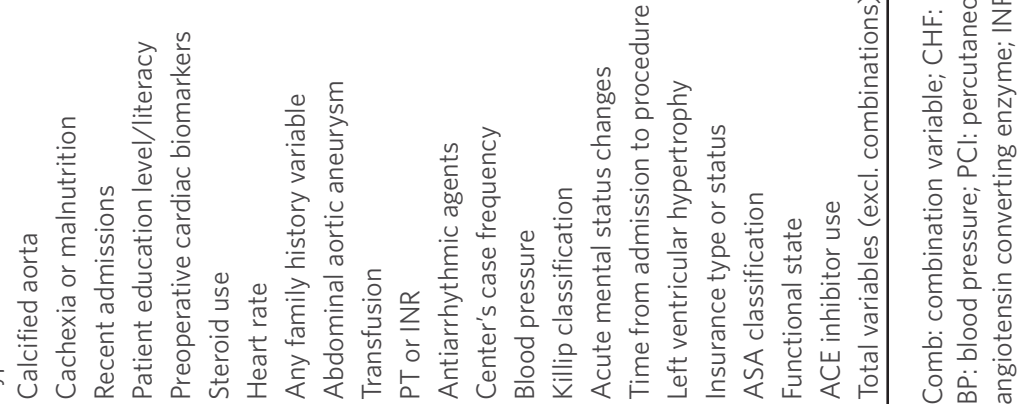

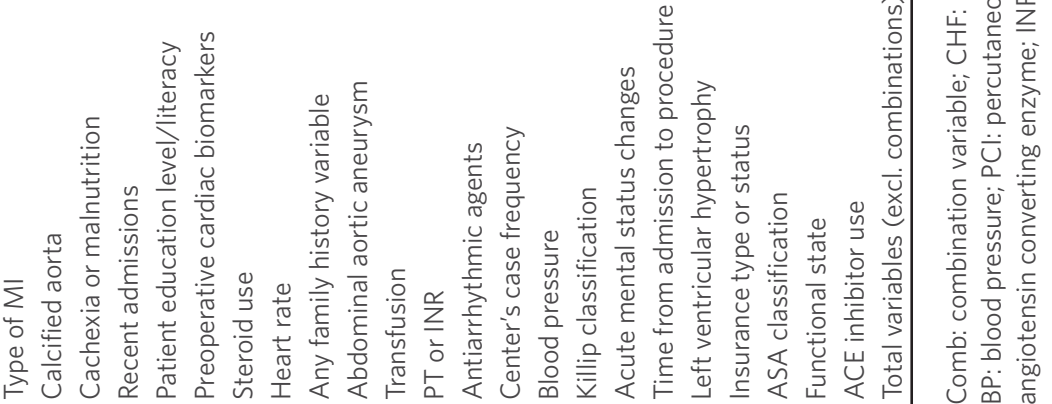

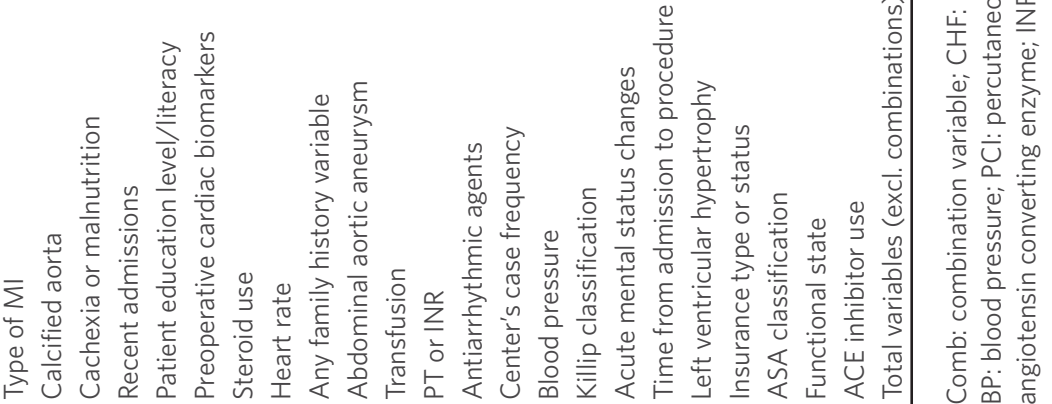

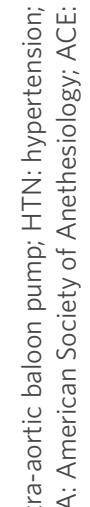

童芒

要竞

ह

은

要

$\frac{0}{0}$

ن⿺ 윔

들

离

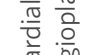

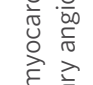

$\vec{E}$
$\ddot{\bar{v}}$
$\dot{\bar{\Sigma}}$

동

.

莲亭

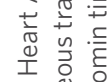

总焉旁

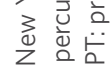

茫芯兽

产言旁

要递

节产高

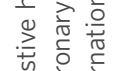

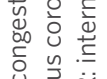

它号:

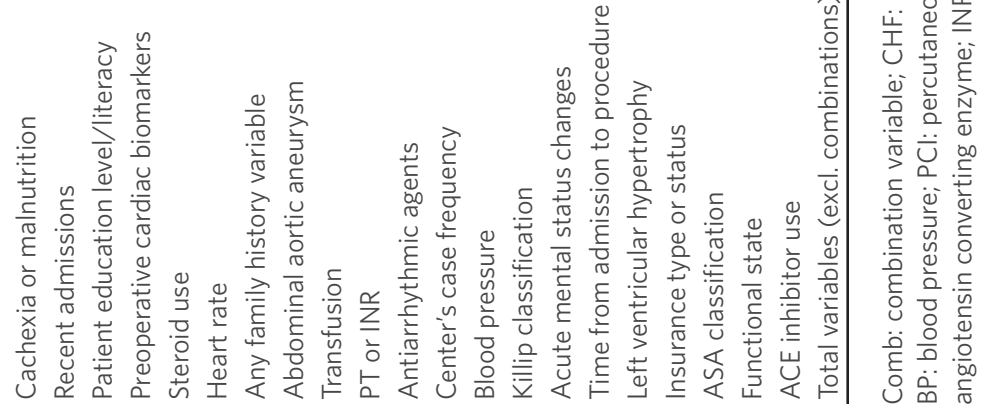

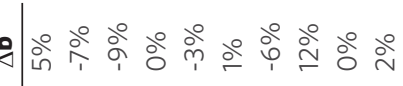

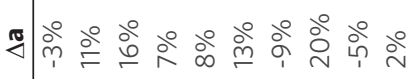

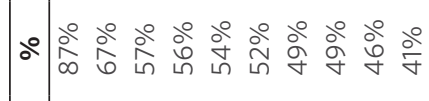

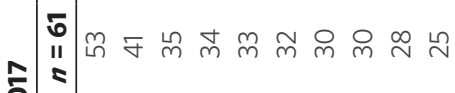

울

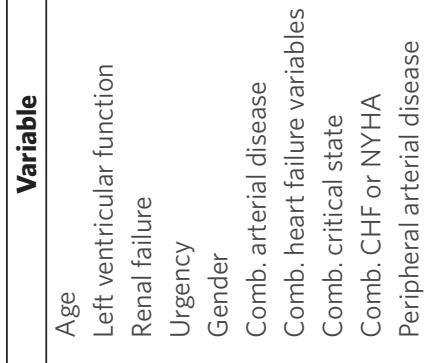

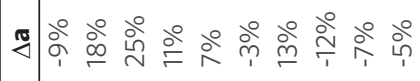

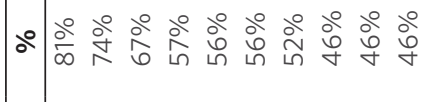

กิ

"I $\forall$ 우

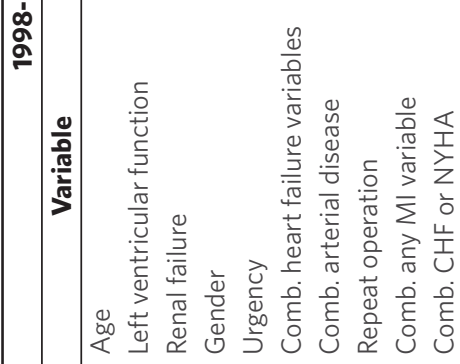

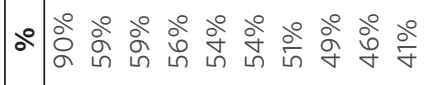

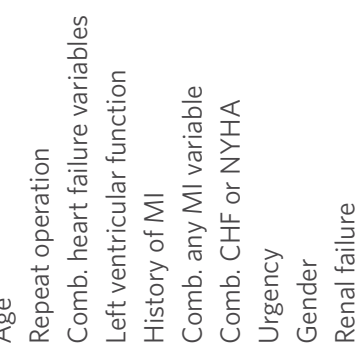




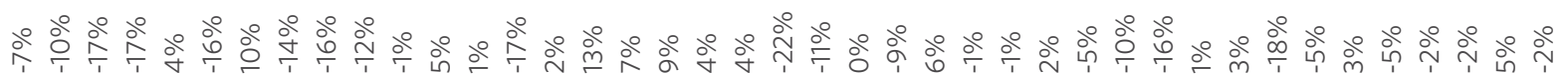

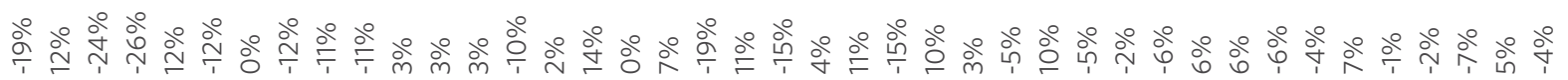

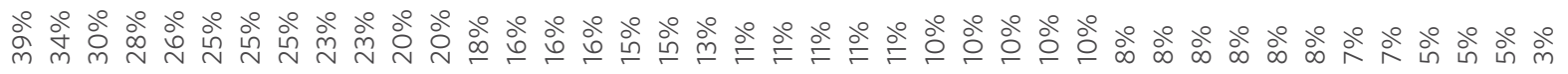
ปั

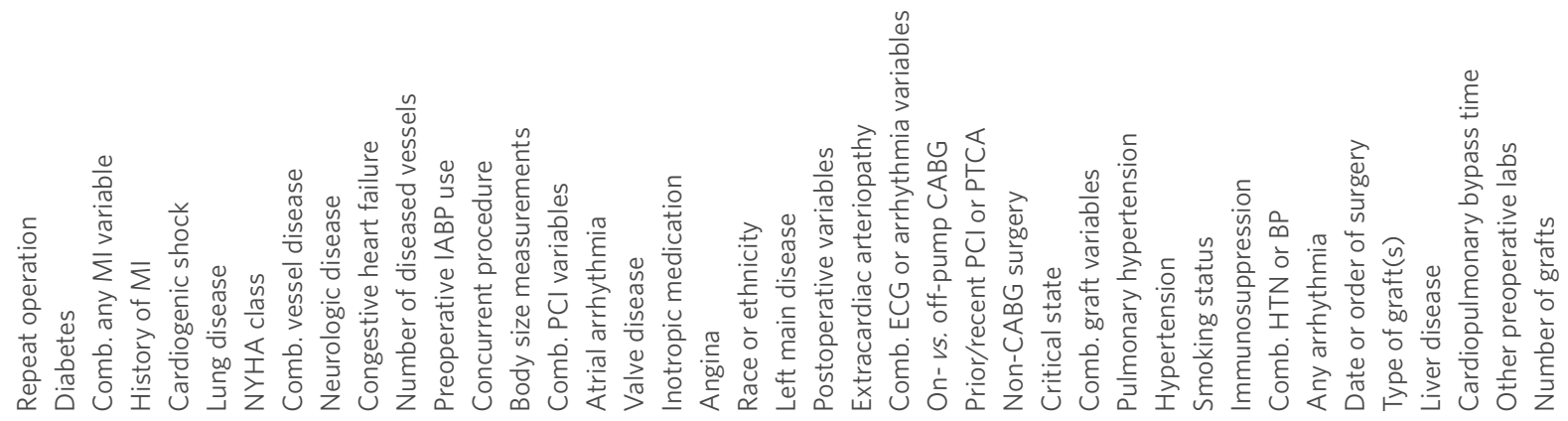

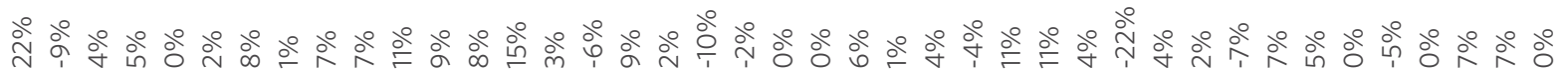

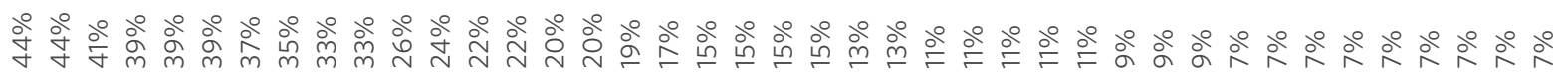

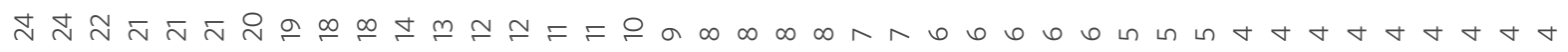

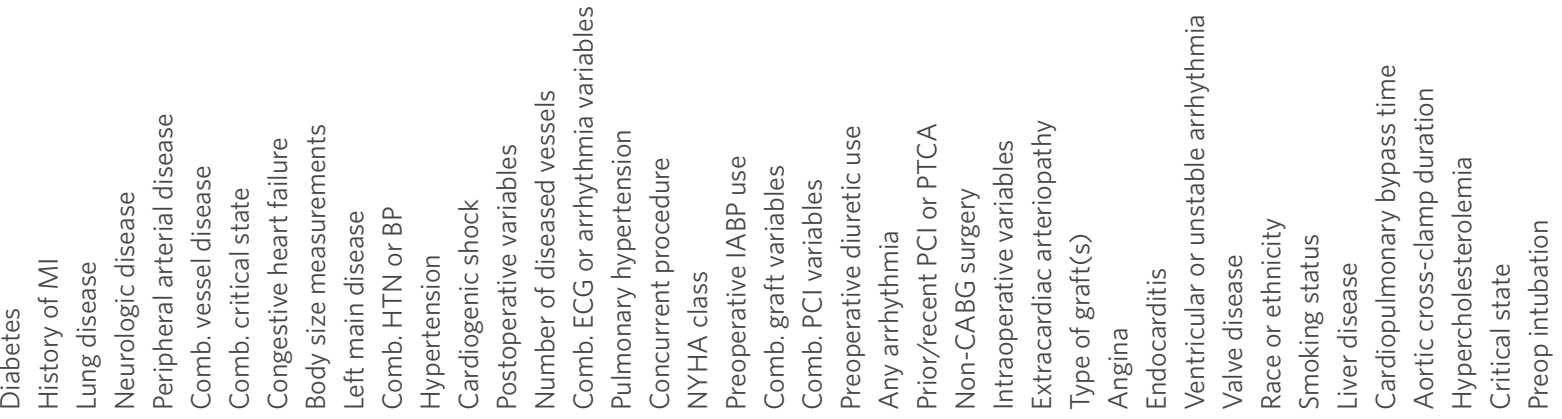

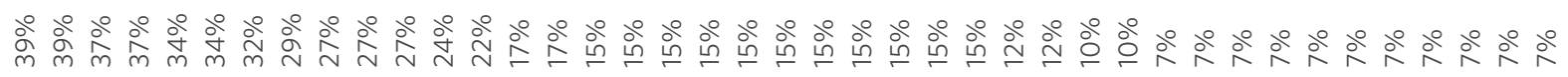

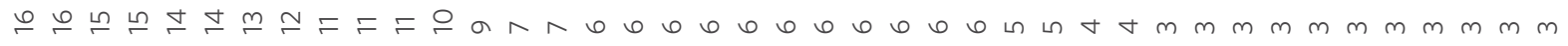

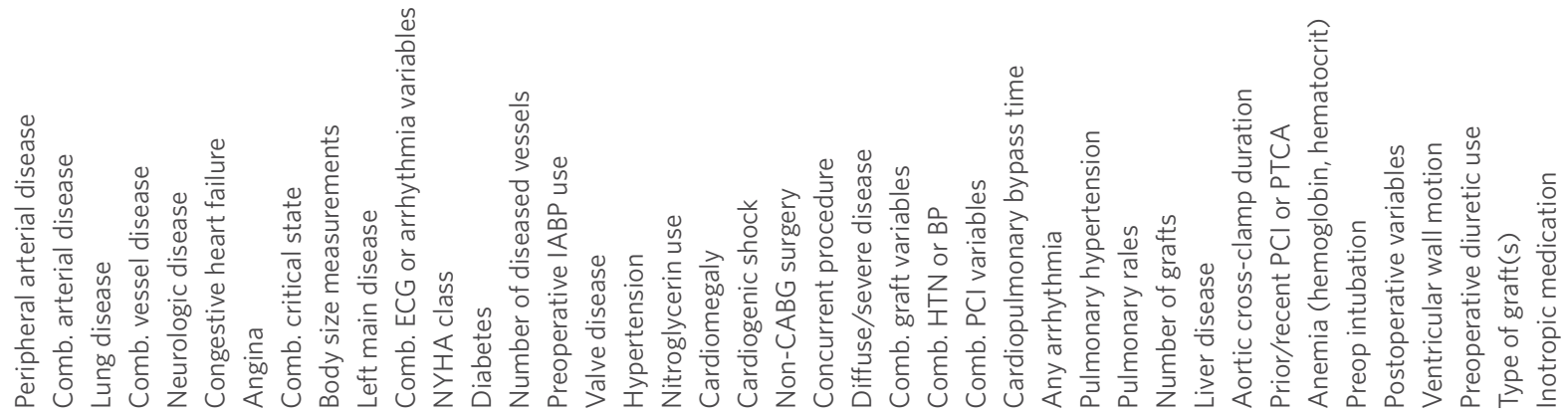




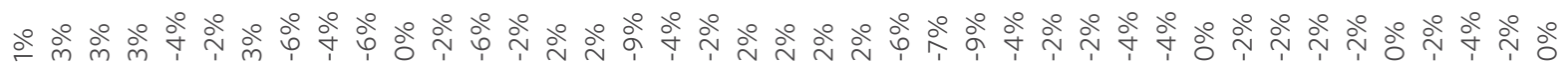

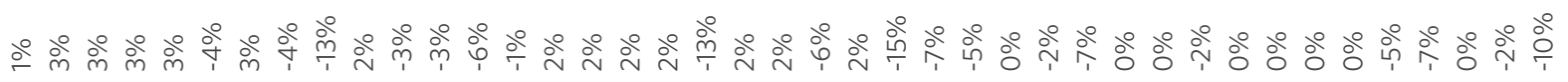

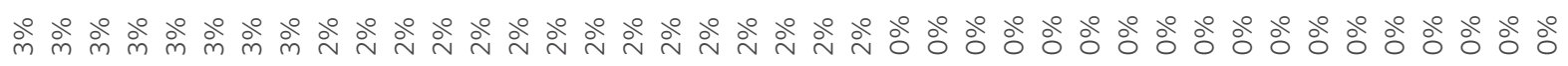

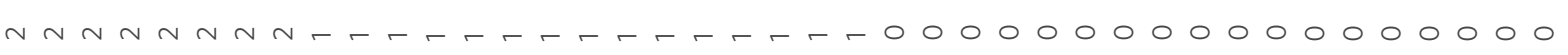
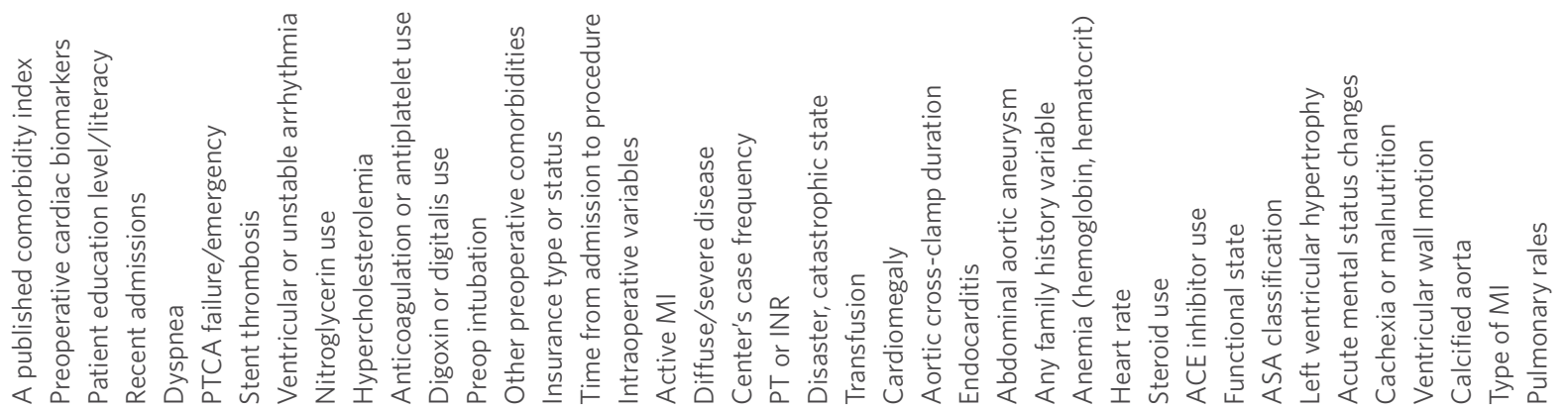

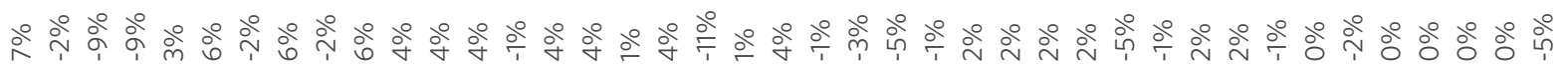

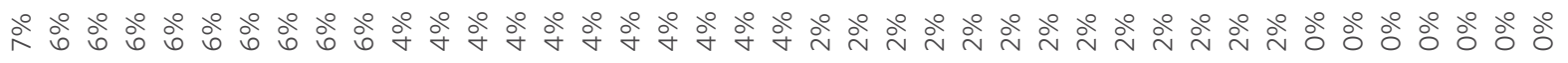

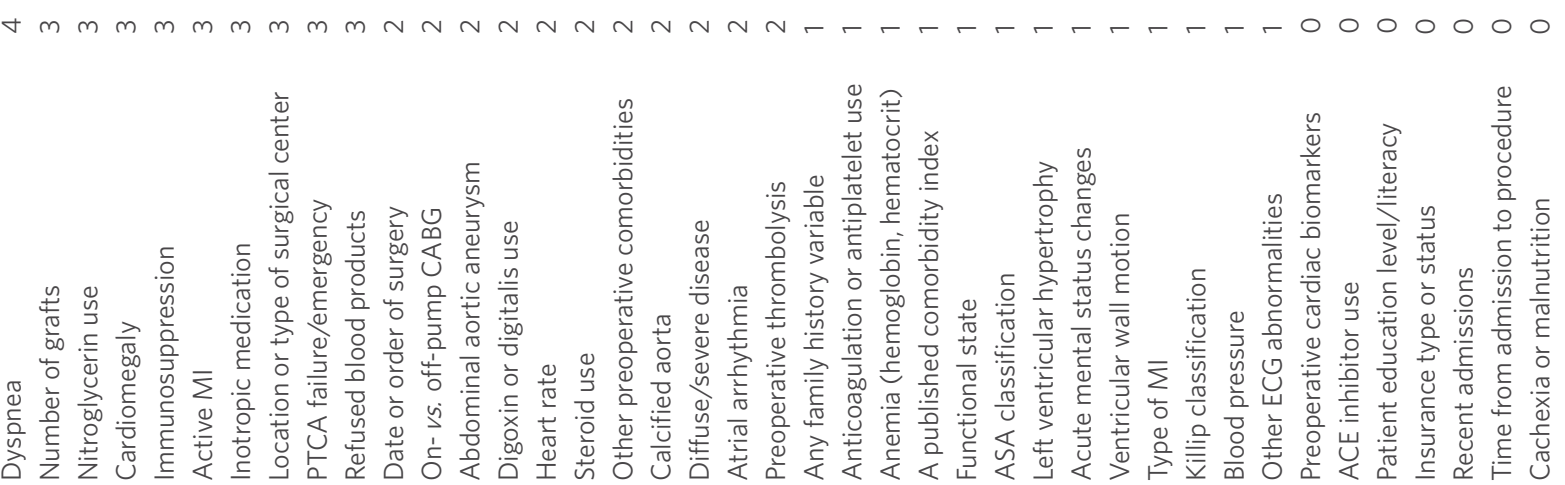

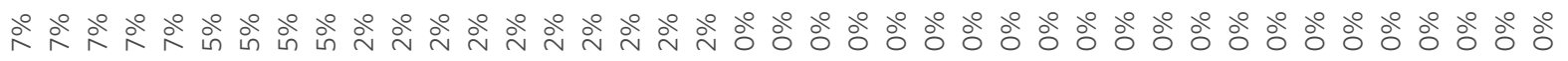
$m m m m m n n n n+\ldots+\ldots+\ldots-n 000000000000000000000$

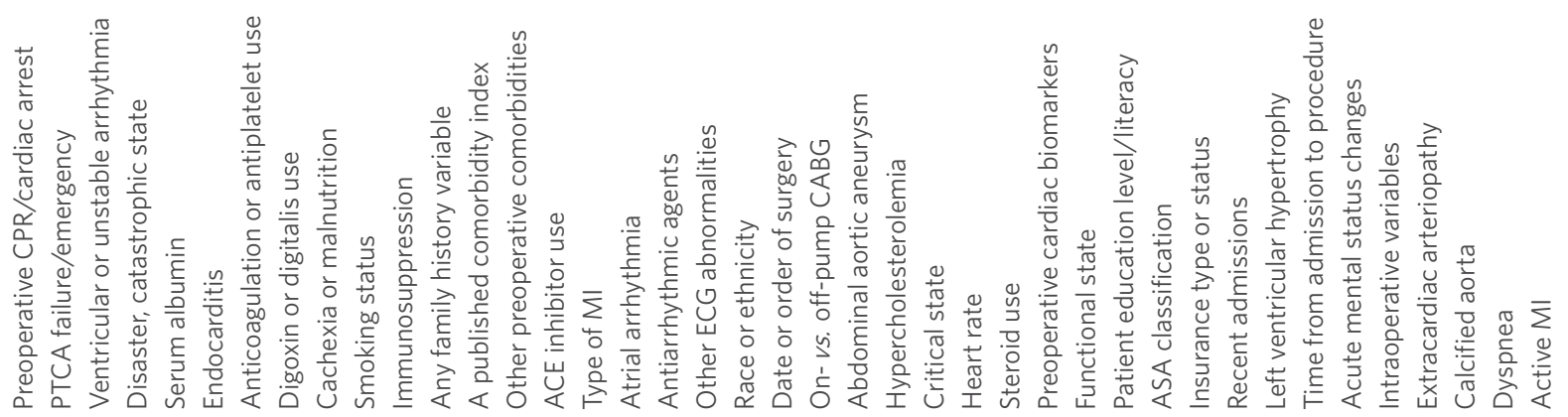


Page 10 of 21

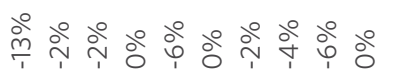

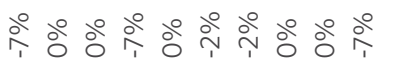

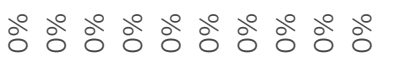

0000000000 t

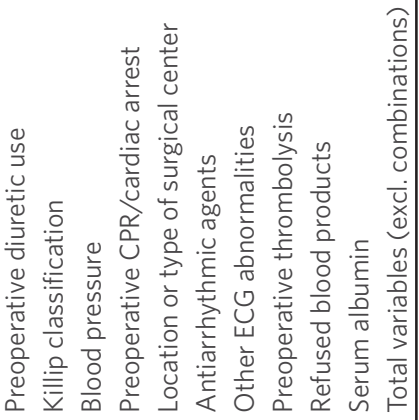

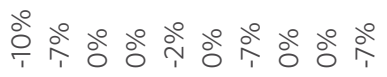

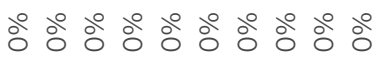

0000000000 요

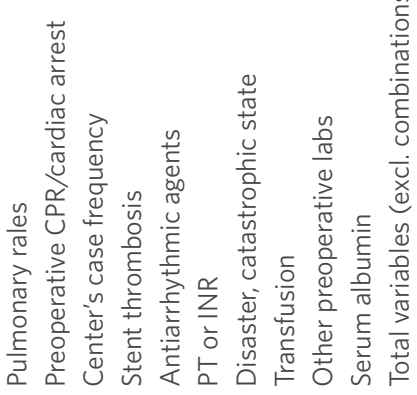

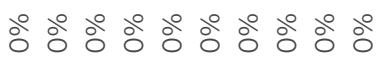

$0000000000 \%$

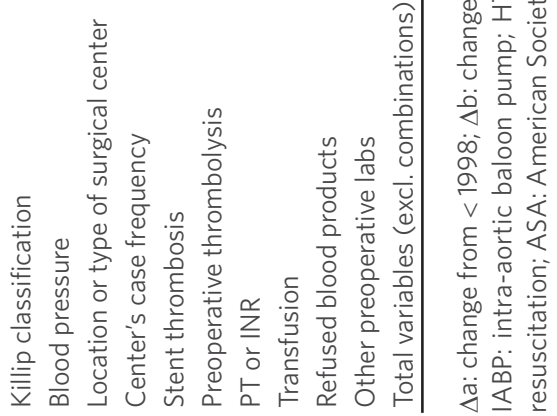

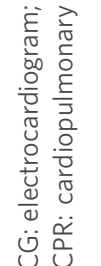

证

芒空

竞 $\frac{0}{5}$

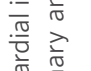

है ㅎํำ

$\dot{\bar{\Sigma}} \stackrel{\bar{\pi}}{\frac{\pi}{5}}$

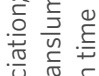

पू

这呇旁

西完

产竞

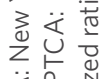

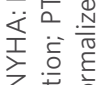

$\sum_{i=\frac{1}{0}}$

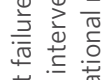

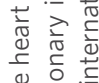

空高妾

ob o d

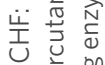

产 㐫.

迹高

드 힐

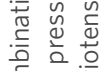

등 믕 है

U

응 은 은

它

定全

일

ฮै हैं

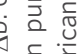

के 응

要远

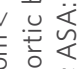

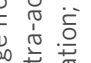

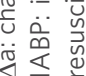

Carr et al. Vessel Plus 2020;4:12 I http://dx.doi.org/10.20517/2574-1209.2020.01

T)

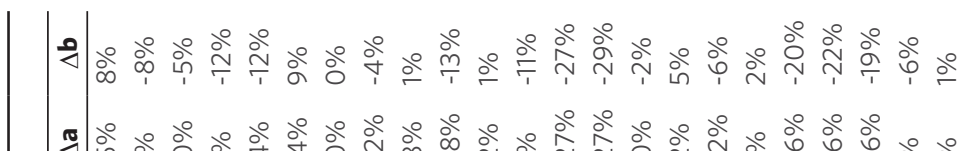
ๆ

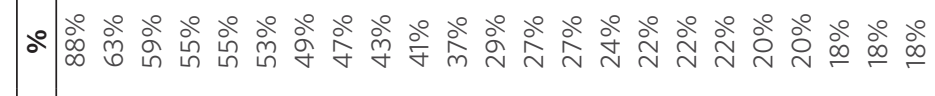

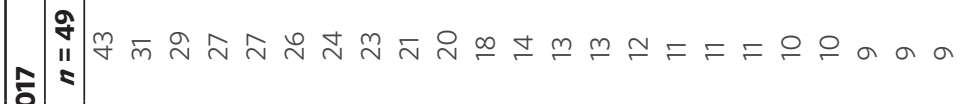

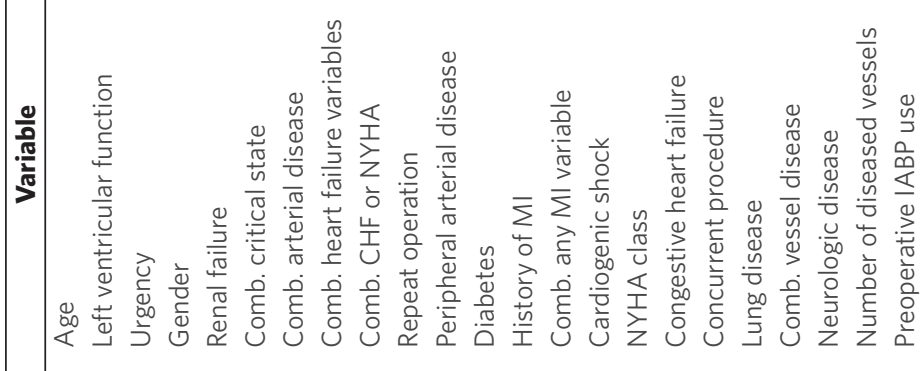

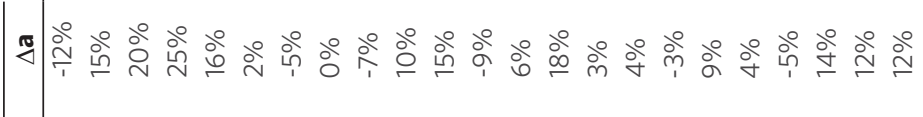

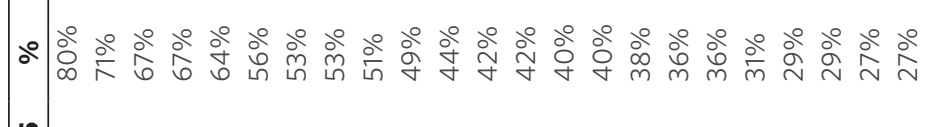

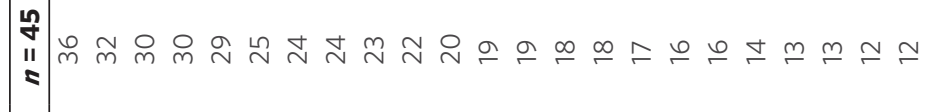

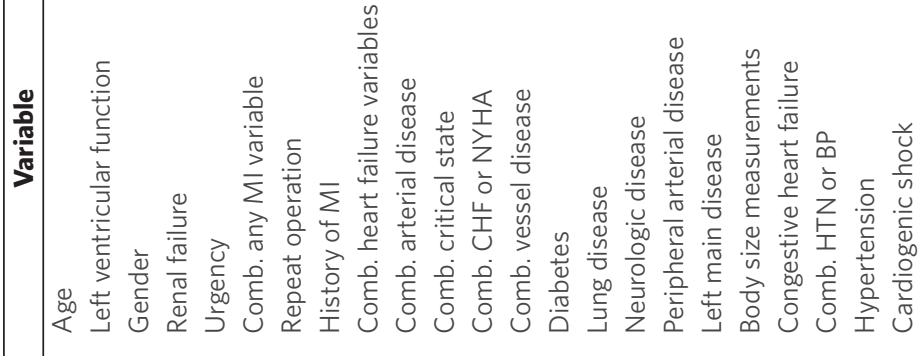

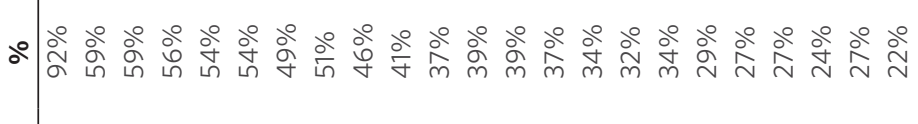

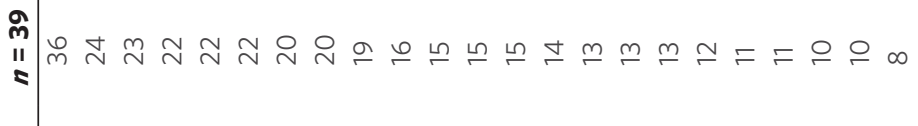

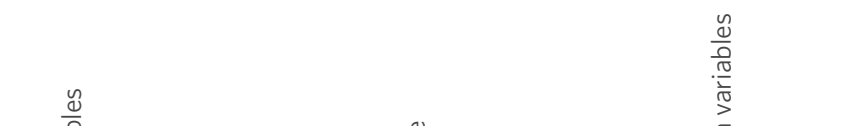

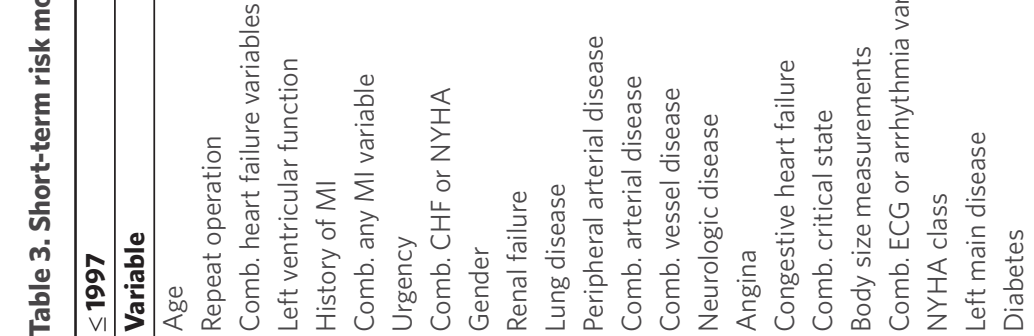




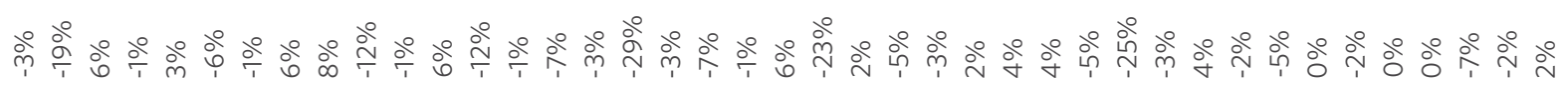

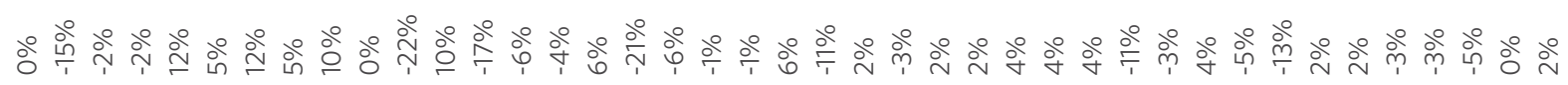

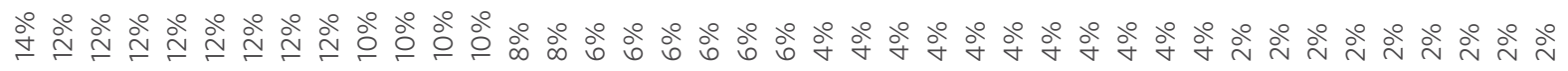

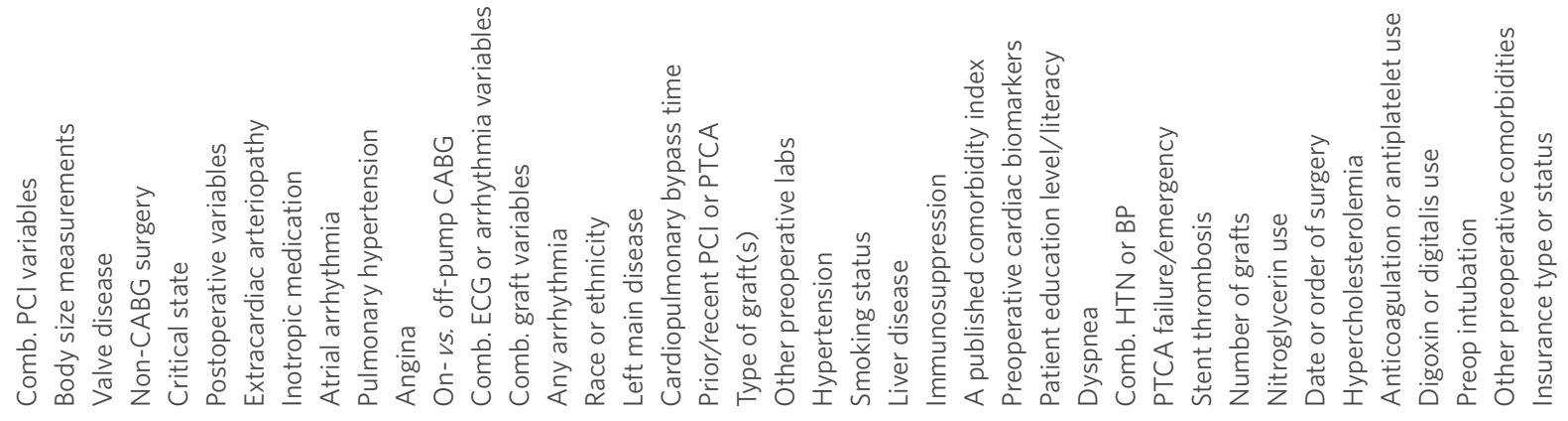

옷ํํ 운

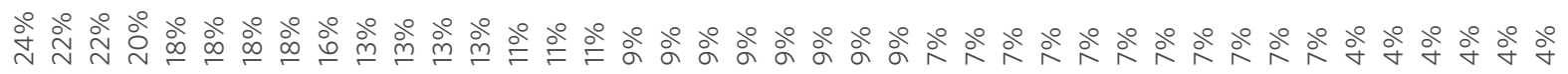

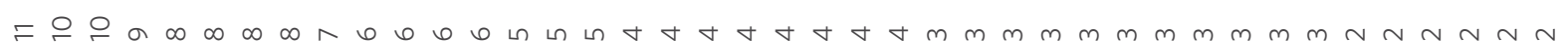

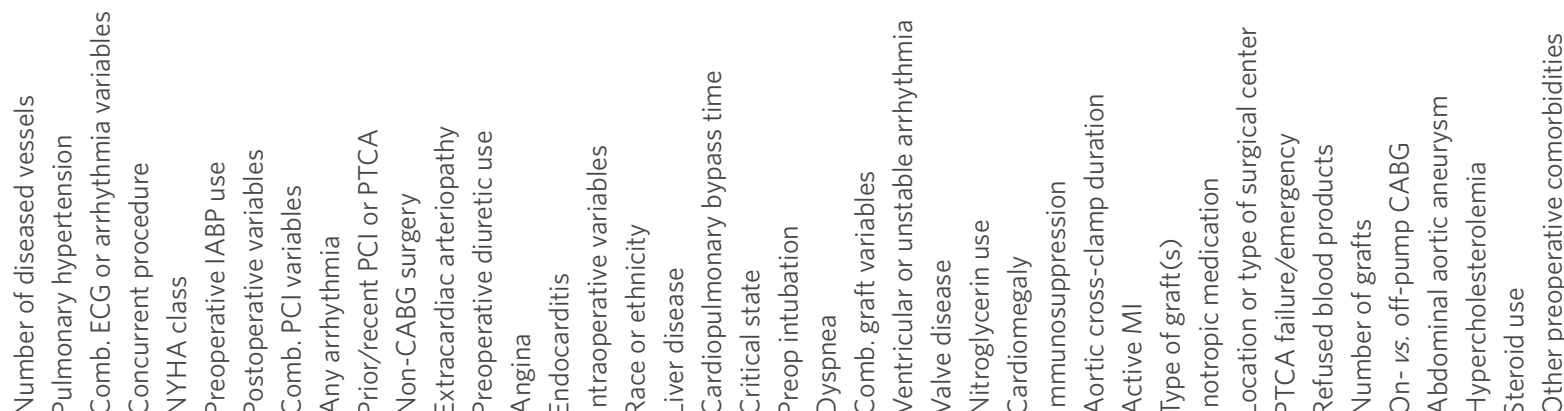

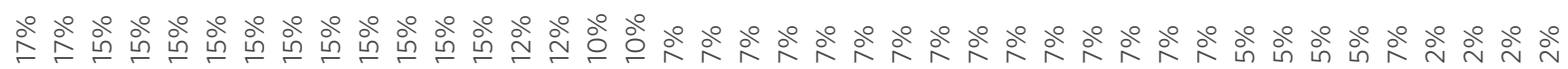

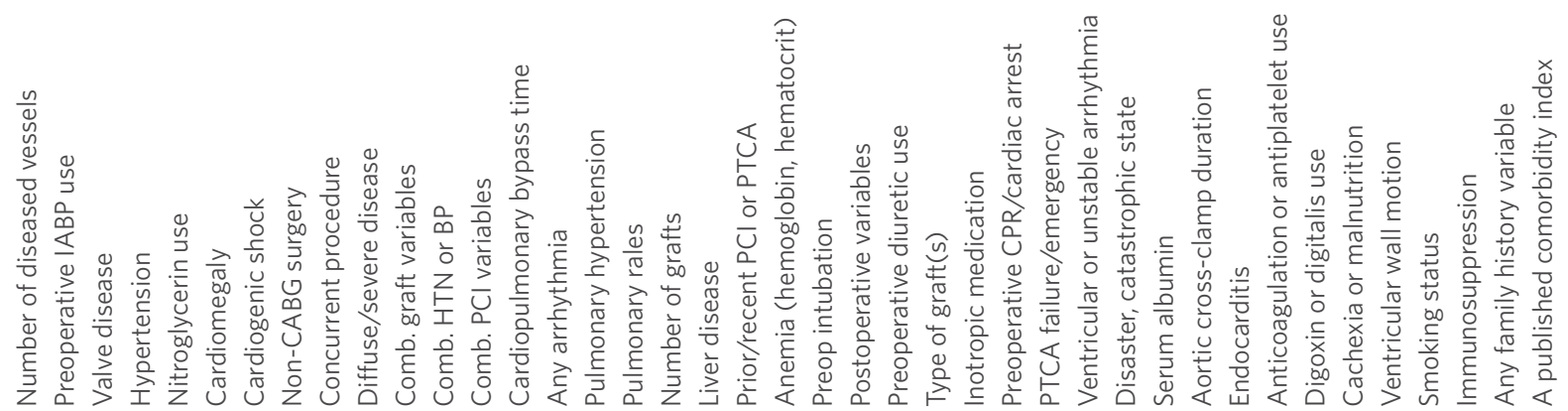




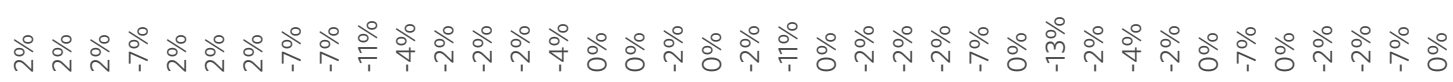

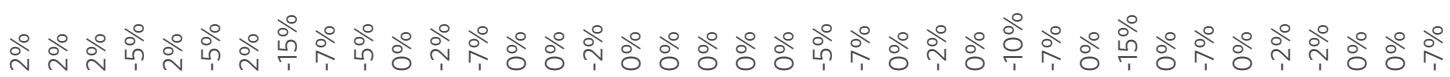

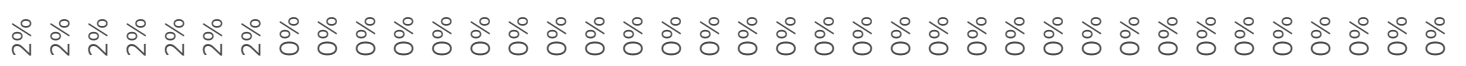
$\ldots+\ldots 0000000000000000000000000000000 \overline{0}$

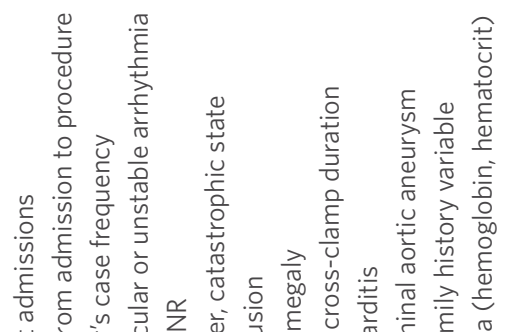

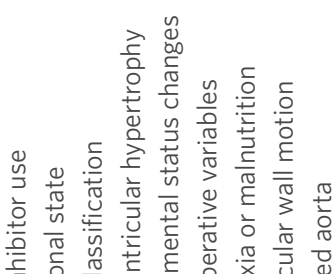

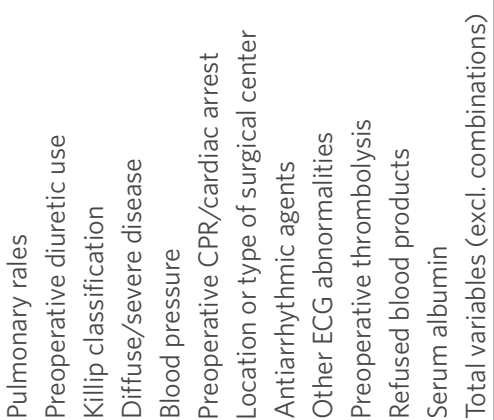

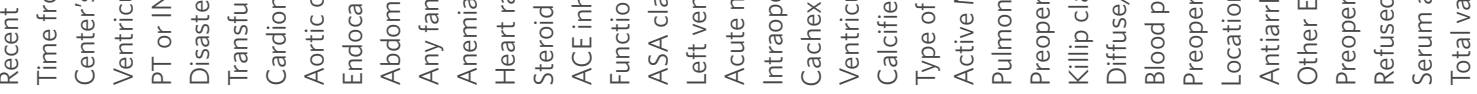

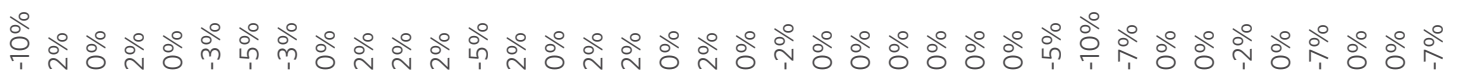

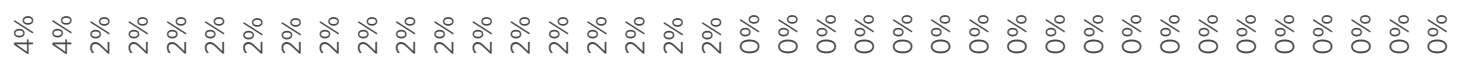

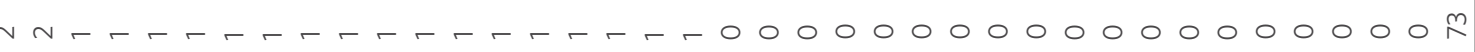

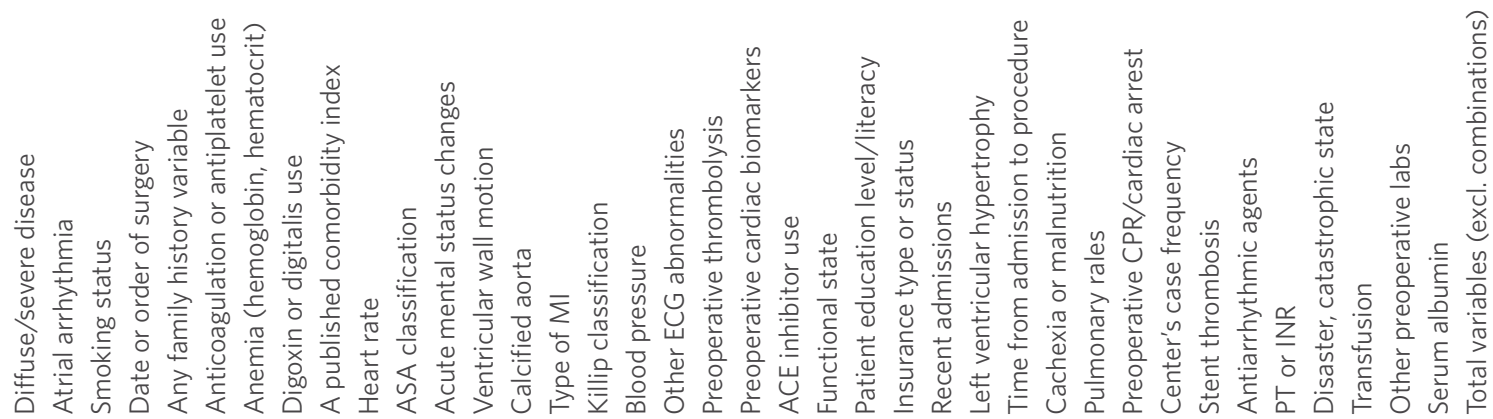

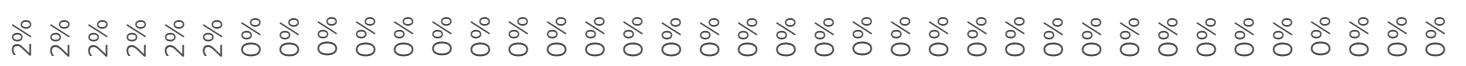
- - - - $00000000000000000000000000000000 \%$

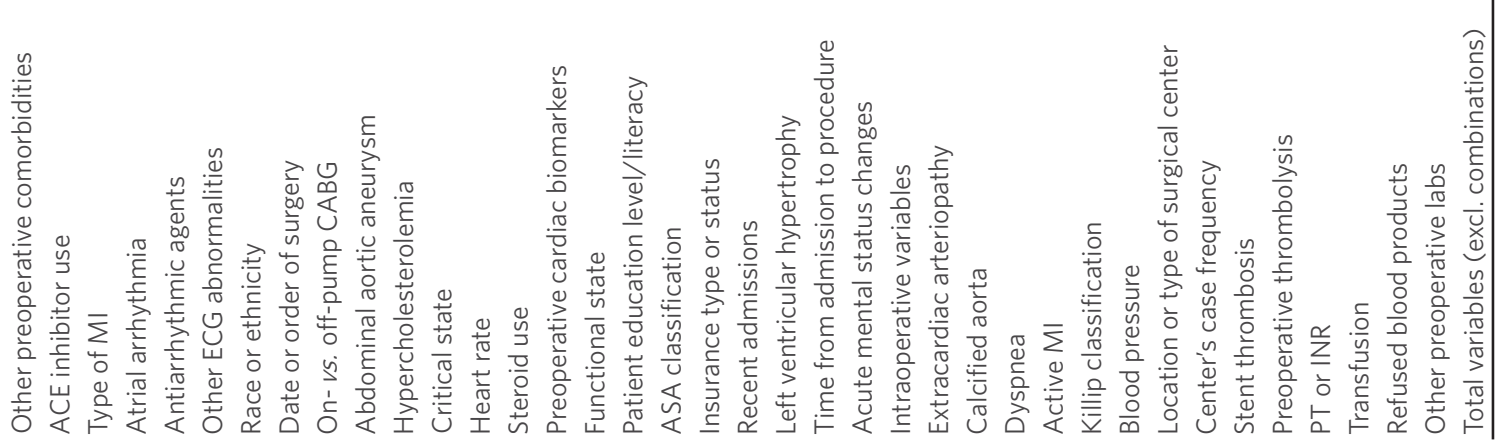

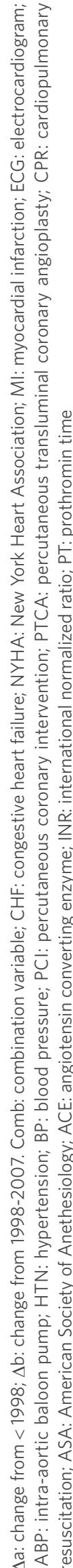




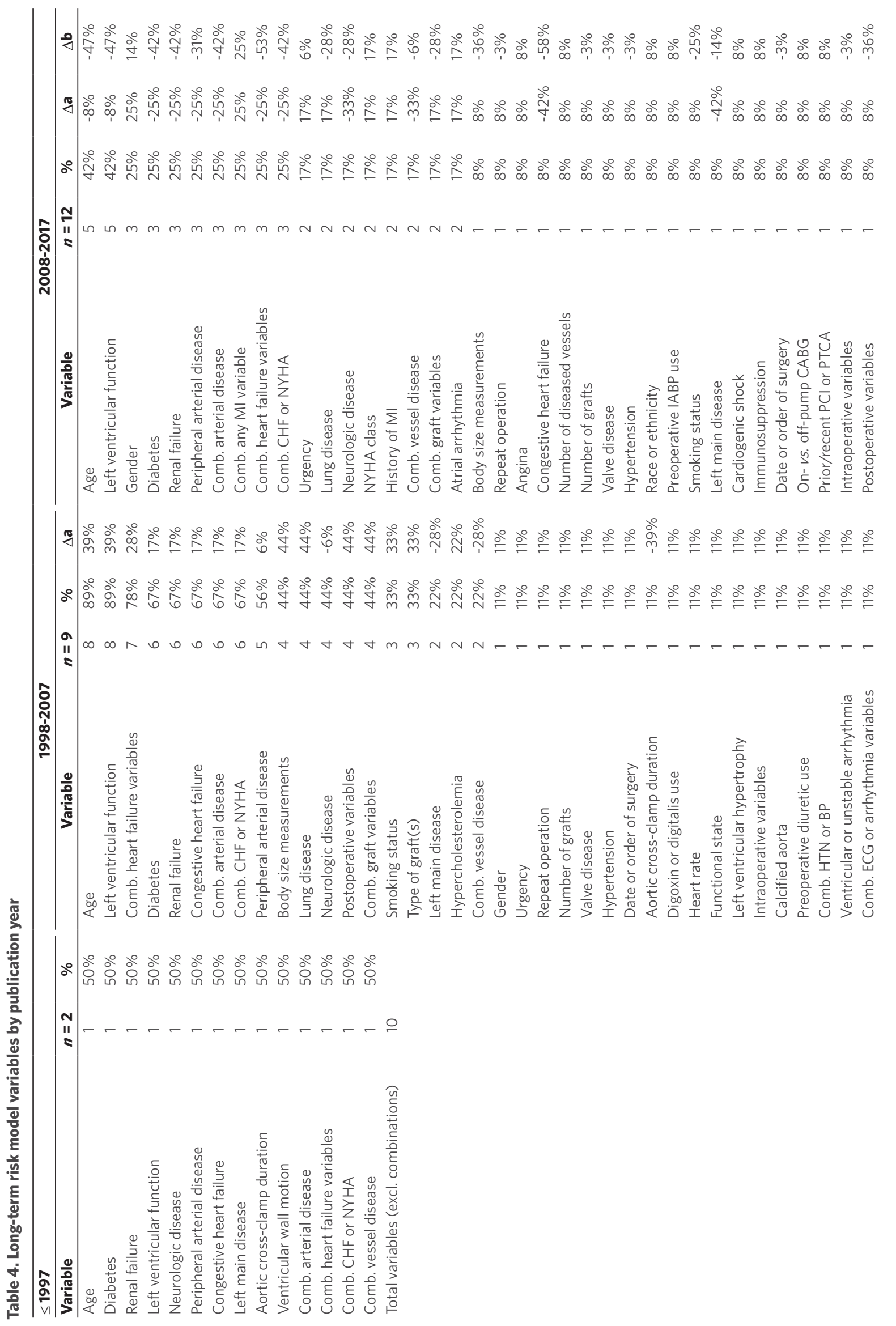




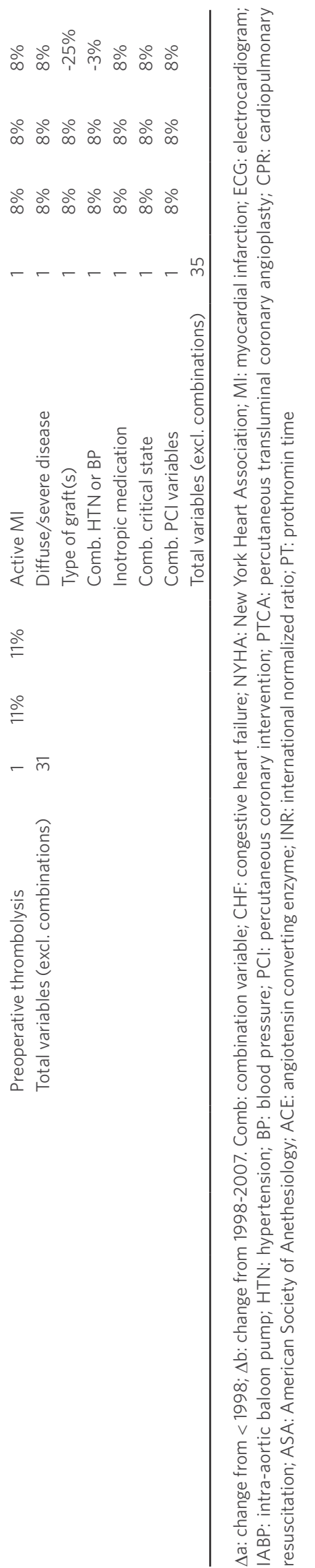

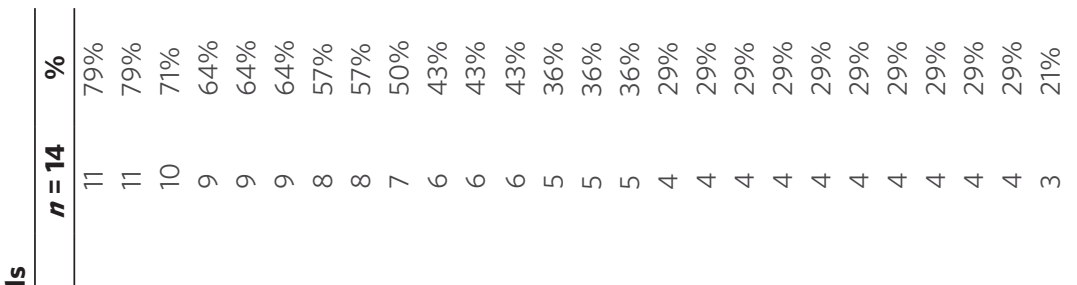

$\frac{n}{\frac{0}{0}}$

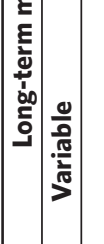

垔

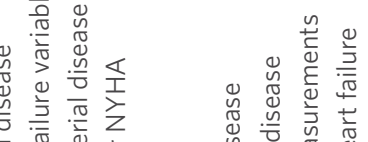

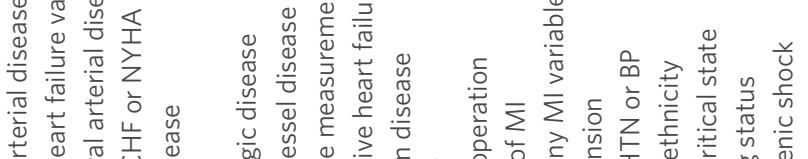

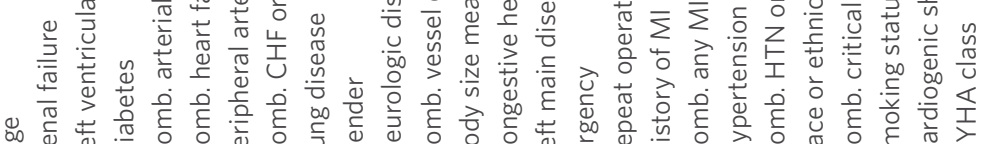

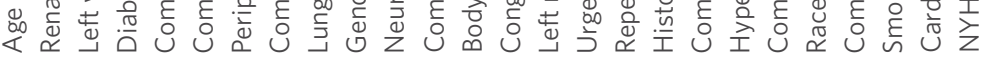

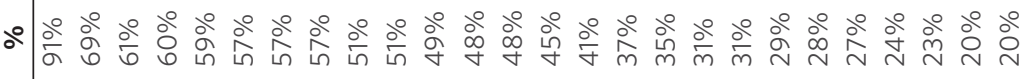
ผ

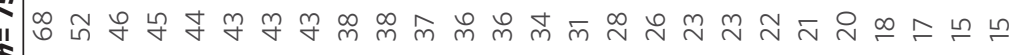

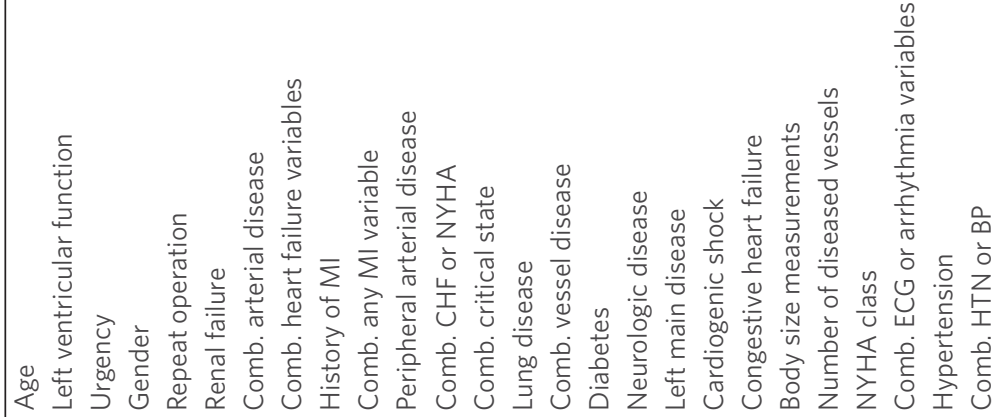

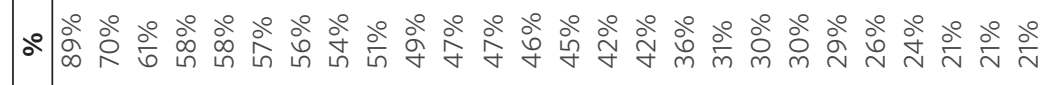

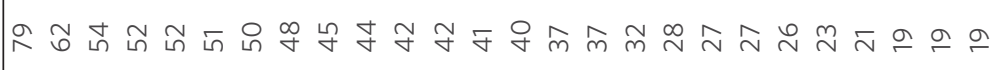

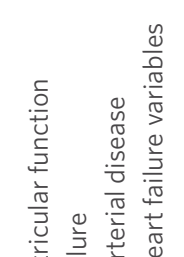

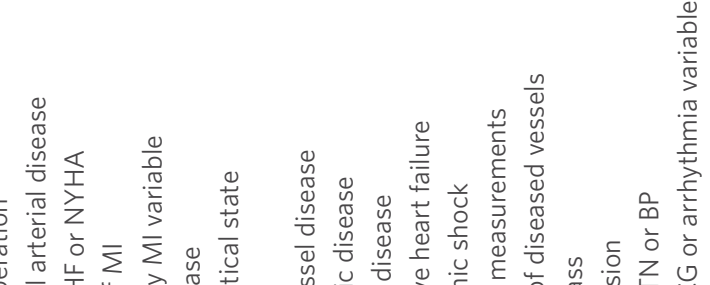

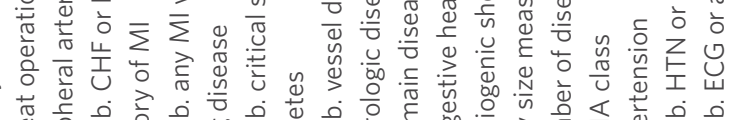

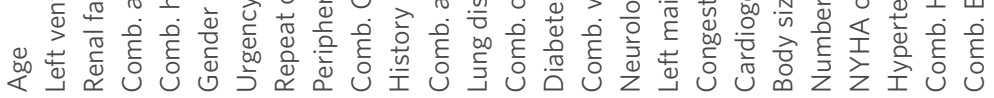




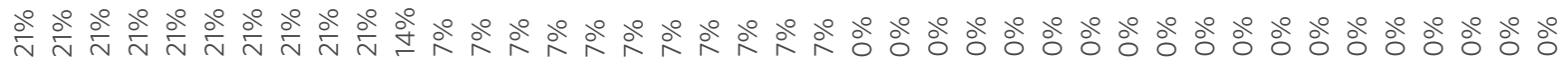

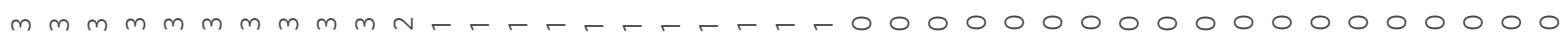

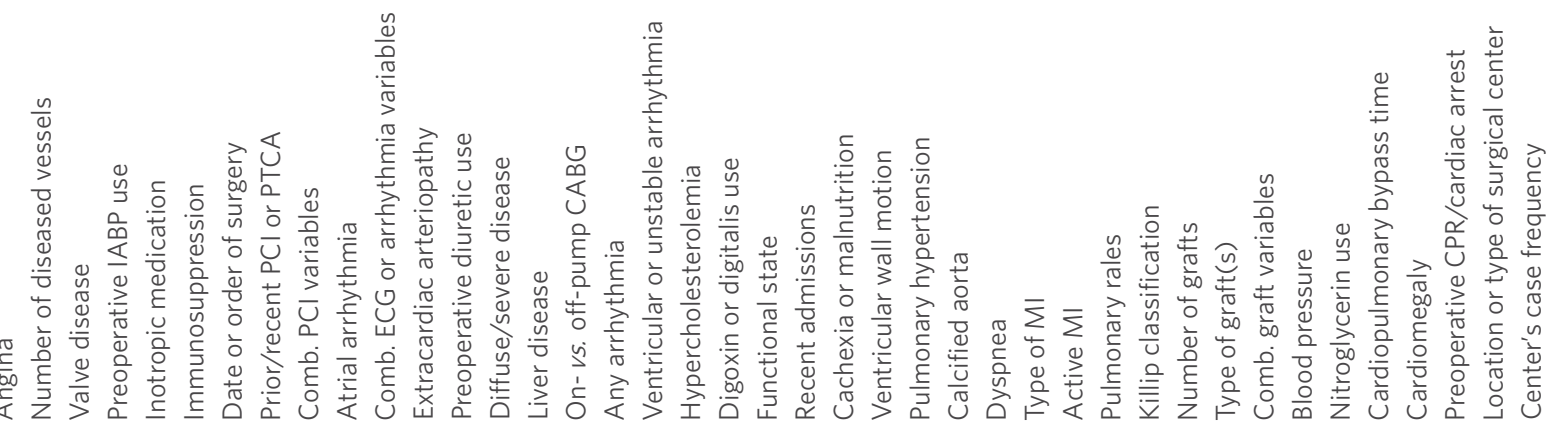

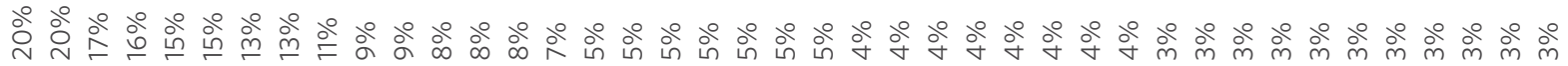

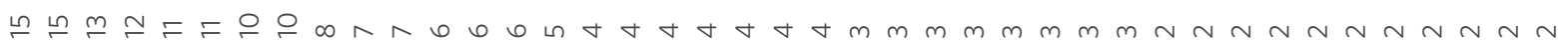

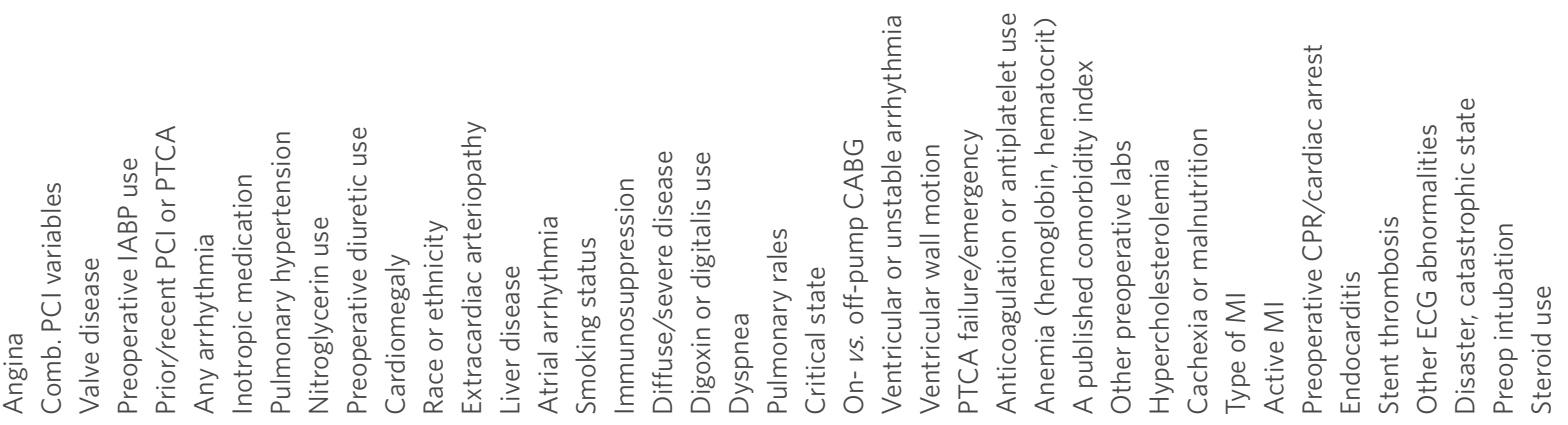

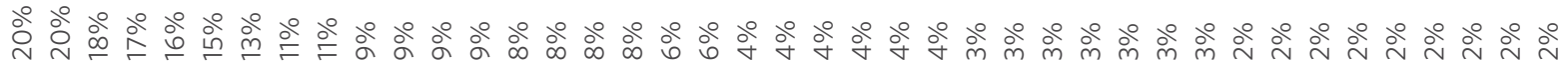

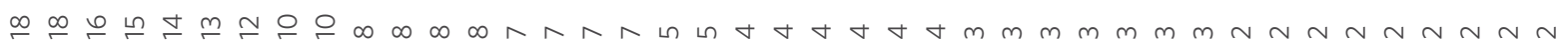

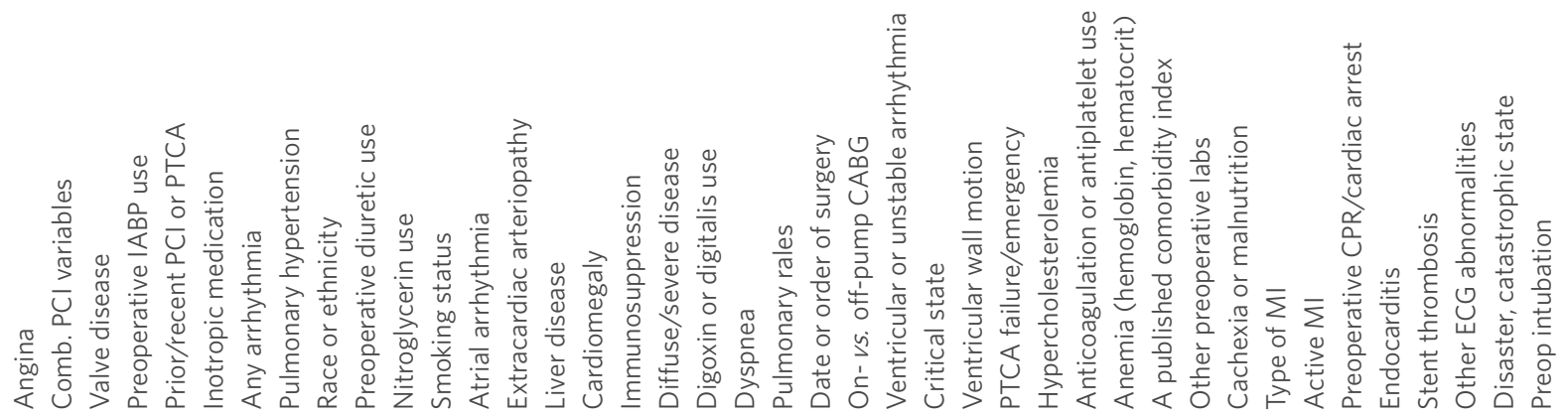




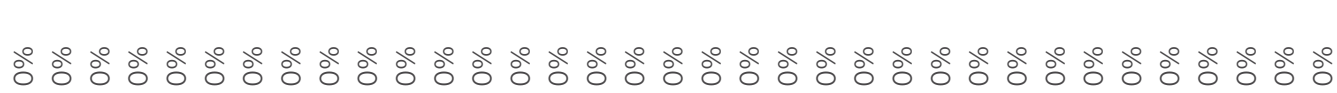
$00000000000000000000000000000000000 \mathrm{~m}$

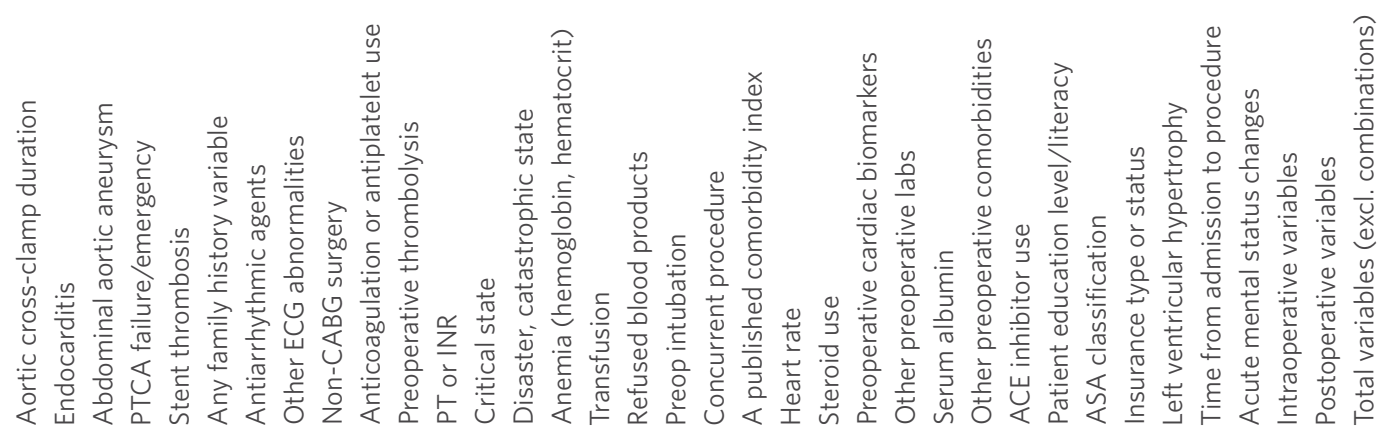

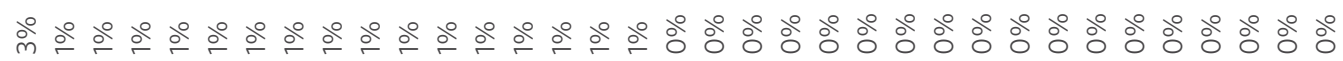

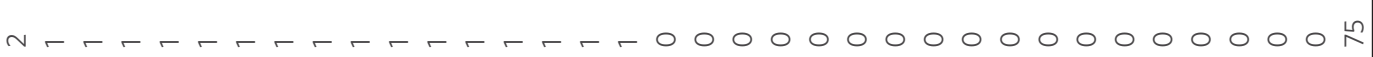

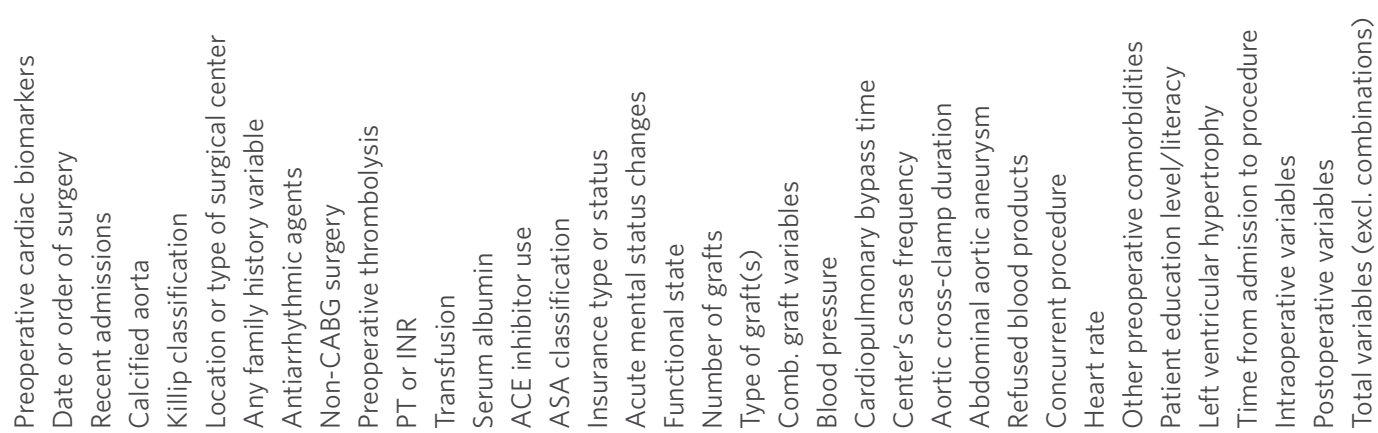

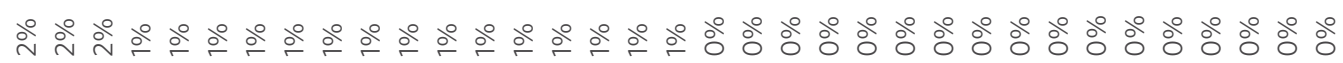

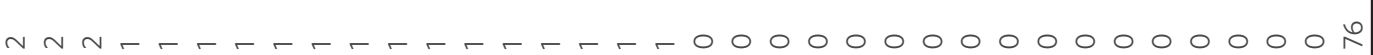

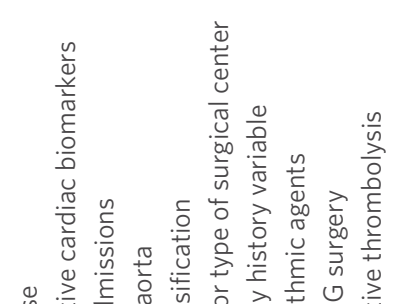




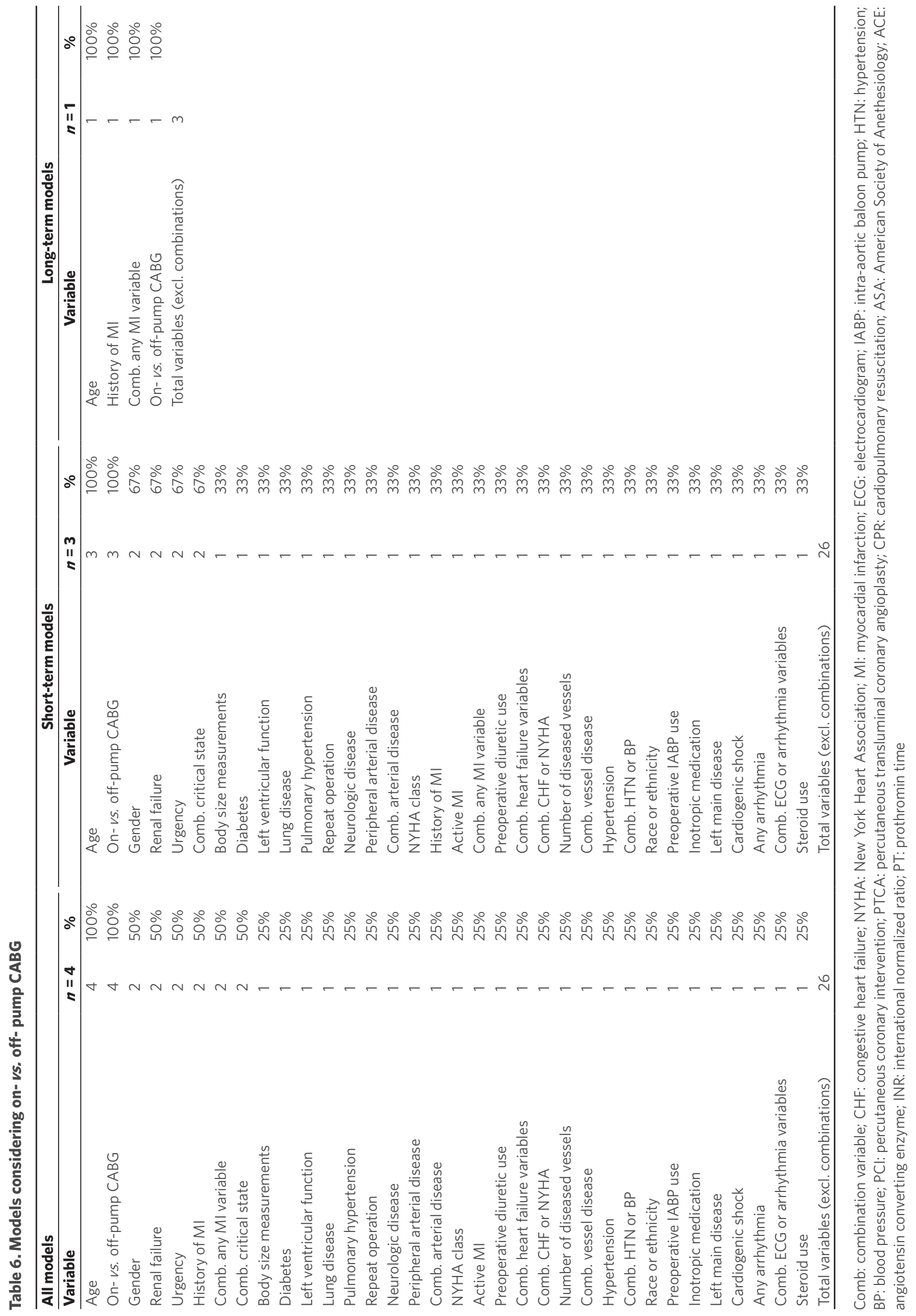


minor differences in the pre-CABG patients' risk factor frequency (which may have been associated with provider-based off-pump patient selection criteria), the pre-CABG patient risk factors identified were extremely similar to the overall findings, as reported above. Given the smaller number of on-pump vs. off-pump CABG mortality risk model comparisons reported, however, these findings may have limited generalizability.

When reviewing the frequency distribution of preoperative model risk variables, it is striking how very few modifiable (as opposed to non-modifiable) patient risk factors have been identified with a post-CABG mortality impact. As an inherently non-modifiable risk factor, the risk for post-CABG mortality increases as a patient's age increases. Perhaps by the time a patient is being evaluated for a CABG procedure, the negative prognostic impact for the most common preoperative risk factors, such as diabetes mellitus and poor left ventricular ejection fraction, may be difficult to reverse or otherwise counteract in the ST; however, these impacts can be seen in LT models.

In contrast, several of these reported patient risk factors have potential to be mitigated. As an example, body mass index or another marker of body habitus (e.g., height, weight, or body surface area) was included in 31/133 (23\%) of ST models considering only preoperative risk factors. Similarly, a measure of smoking or tobacco use was considered in only $4 / 133$ (3\%). Although it is a well-known fact that these 2 risk factors represent important drivers for a patient developing ischemic heart disease, their significance in predicting post-CABG mortality risk appears likely confounded with presence of diabetes mellitus and poor renal function, which may also be sequela of obesity or diabetes.

Although these risk models may be helpful to enhance the providers' discussions with patients during the informed consent process or support provider discussions as to treatment-related risks for adverse events, the currently published CABG mortality risk models fall short of providing clinicians with useful information to optimize postoperative care consults, to ensure continuity of post-discharge care, or to enhance LT patients' survival. While it would likely not be surprising to most clinicians that these modifiable risk factors are important considerations, the manner presented in LT risks models may give the impression that LT post-CABG mortality risk is set in stone at the time of surgery, rather than an evolving risk that can be mitigated or exacerbated at any time. Using follow-up time-period-based risks (e.g., hemoglobin A1c management or continued tobacco use), therefore, future sequential modeling approaches may be needed to help better guide post-CABG follow-up care decisions and to optimize LT post-CABG survival.

One risk factor that is potentially modifiable, but not in the traditional sense, is operative urgency or priority, meaning whether a given procedure was performed in the elective $v s$. urgent or even emergent manner with an unstable patient. As clinically relevant examples, it is important to know when to intervene in patients with active angina or acute myocardial infarction. While operating in a time sensitive manner under potentially suboptimal conditions may be unavoidable, the fact that priority or status variables have been identified so frequently as ST mortality risk factors would suggest that future research funding should be prioritized to evaluate the impact of differential pre-CABG waiting periods ${ }^{[16]}$.

A limited number of CABG mortality models found preoperative medications such as nitrates, anti-platelet agents, angiotensin converting enzyme inhibitor, or anti-arrhythmic medication were associated with mortality. Given risk assessment inconsistencies, some of these medications (e.g., nitrates) may have been markers for the severity of coronary disease or preoperative instability. Other medications may, in fact, be markers of optimal medical management during the pre- and postoperative periods ${ }^{[17]}$. 
Currently, no risk models incorporate direct measures of adherence with published clinical practice guidelines (e.g., the American College of Cardiology's guidelines for treatment of coronary artery disease) such as documenting the use of ischemic heart disease medications (e.g., pre-CABG statin use). As a potentially novel and important future enhancement to preoperative risk stratification, adherence to published guidelines should be considered. In general, adherence with published guidelines are increasingly becoming a marker used to identify high-quality, high-value care providers. Adherence to published guidelines has been shown to be suboptimal after CABG, yet adherence has been repeatedly associated with improved cardiovascular-related mortality in various populations ${ }^{[18-20]}$. Applied proactively, guideline adherence may provide a useful direction for future cardiac surgery mortality risk modeling endeavors.

Interestingly, none of these CABG mortality risk models identified mental health-related (e.g., psychiatric) or socioeconomic risk factors as predictive; however, preoperative depression has been associated with increased 5- and 10-year post-CABG mortality ${ }^{[21,22]}$. Similarly, one recent study showed a community-based marker of socioeconomic status (e.g., the Distressed Community Index) to be predictive of in-hospital mortality ${ }^{[23]}$. Hence, these types of non-traditional CABG risk factors may be worthy of future exploration.

\section{Limitations}

Conducted as an advanced PubMed literature review in February 2019, this summary has identified knowledge "gaps", which are intended to foster future CABG risk modeling research. With collaborative team member oversight and guidance, the majority of these data extractions were performed by a single author (BC). Substantial overlap was documented among several risk variables (e.g., left ventricular ejection fraction $v s$. congestive heart failure $v s$. pulmonary rales $v s$. diuretic use); therefore, the relative impact of any individual risk factor could not be easily quantified. If standardized CABG quality improvement database definitions (e.g., the Society of Thoracic Surgeons' definitions) were uniformly utilized in the future, however, comparing variable-specific relative rankings (e.g., identifying the "top five variables impacting mortality" across all published models) would become possible.

Inherently, all risk variables reported were limited to the sub-group of patients' risk characteristics uniquely captured by each database. Although a common core of risk variables was captured, each dataset may have contained unique risk factors relevant specifically to their patient populations. Additionally, different risk modeling approaches (e.g., descending stepwise logistic regression) may have contributed to the variations documented for the risk factors associated with post-CABG mortality.

In conclusion, $\mathrm{CABG}$ maintains an important role in the management of coronary artery disease; thus, understanding patients' ST and LT surgical risk and risk factors remains important to optimizing CABG patient's selection, treatment, and follow-up care. A wide array of CABG mortality model findings and an equally vast diversity of analytic approaches were used, each prediction model having population-specific benefits and drawbacks. Over the past 20 years, it appears that the majority of CABG registries have come to a general consensus to utilize at least a core pre-CABG risk factor set. Beyond this core dataset, however, population-relevant risk factors are commonly reported.

As always, research continues to identify new risk factors that may affect post-CABG patients' risk; based on these data-driven findings, areas warranting further research were identified - such as incorporating modifiable risk factors and ischemic heart disease guideline compliance. Additionally, a new focus appears warranted to evaluate pre-CABG wait time impacts upon surgical priority, as well as CABG risk-adjusted outcomes. Applying the lessons learned, post-CABG mortality risk model findings may be quite different in the future from current findings - as the post-CABG care continues to improve and the field of statistical risk modeling advances forward. 


\section{DECLARATIONS}

\section{Authors' contributions}

Wrote the initial study protocol, under the oversight and leadership of Grover FL: Carr BM, Shroyer ALW Prepared the research-related materials to obtain an official determination of "not research" by the Northport VA Medical Center's Research and Development office: Shroyer ALW

Performed the detailed data after implementing the advanced literature search strategy, acquisition with active involvement by Grover FL and Shroyer ALW: Carr BM

Ran the initial data analyses and prepared the initial set of tables and figures: Carr BM

Aided in the interpretation as well as the full co-author team worked collaboratively to assure a comprehensive search strategy: Grover FL, Shroyer ALW

The first draft of this article was written jointly by Carr BM and Shroyer ALW, with revisions provided by Grover FL, all co-authors provided their final approval.

\section{Availability of data and materials}

This study's data file, including data extracted for each reference listed, is available as an online-only supplement (Appendix A).

\section{Financial support and sponsorship}

None.

\section{Conflicts of interest}

All authors declared that there are no conflicts of interest.

\section{Ethical approval and consent to participate}

The Northport VA Medical Center's Research and Development Office determined that this study was "not research"; this "not research" determination was dated September 12, 2019.

\section{Consent for publication}

Not applicable.

\section{Copyright}

(c) The Author(s) 2020.

\section{REFERENCES}

1. Weiss AJ, Elixhauser A. Trends in Operating Room Procedures in U.S. Hospitals, 2001-2011: Statistical Brief \#171. 2014 Mar. Healthcare Cost and Utilization Project (HCUP) Statistical Briefs [Internet]. Rockville (MD): Agency for Healthcare Research and Quality (US); 2006 Feb. Available from http://www.ncbi.nlm.nih.gov/books/NBK201926/ [Last accessed on 12 Mar 2020]

2. National Heart, Lung, and Blood Institute. Heart Surgery. Available from http://www.nhlbi.nih.gov/health-topics/heart-surgery [Accessed 16 Mar 2020]

3. D'Agostino RS, Jacobs JP, Badhwar V, Fernandez FG, Paone G, et al. The society of thoracic surgeons adult cardiac surgery database: 2018 update on outcomes and quality. Ann Thorac Surg 2018;105:15-23.

4. Grover FL, Hammermeister KE, Burchfiel C. Initial report of the veterans administration preoperative risk assessment study for cardiac surgery. Ann Thorac Surg 1990;50:12-26.

5. Public Law 99-166. Veterans' Administration Health-Care Amendments of 1985. Available from http://www.govinfo.gov/content/pkg/ STATUTE-99/pdf/STATUTE-99-Pg941.pdf\#page=10 [Accessed 16 Mar 2020]

6. Grover FL, Johnson RR, Shroyer AL, Marshall G, Hammermeister KE. The veterans affairs continuous improvement in cardiac surgery study. Ann Thorac Surg 1994;58:1845-51.

7. Jones RH, Hannan EL, Hammermeister KE, Delong ER, O'Connor GT, et al. Identification of preoperative variables needed for risk adjustment of short-term mortality after coronary artery bypass graft surgery. The Working Group Panel on the Cooperative CABG Database Project. J Am Coll Cardiol 1996;28:1478-87.

8. Shroyer AL, Dauber I, Jones RH, Daley J, Grover FL, et al. Provider perceptions in using outcomes data to improve clinical practice. 
Ann Thorac Surg 1994;58:1877-80.

9. Marshall G, Shroyer AL, Grover FL, Hammermeister KE. Bayesian-logit model for risk assessment in coronary artery bypass grafting. Ann Thorac Surg 1994;57:1492-9.

10. Tu JV, Weinstein MC, McNeil BJ, Naylor CD. Predicting mortality after coronary artery bypass surgery: what do artificial neural networks learn? The Steering Committee of the Cardiac Care Network of Ontario. Med Decis Making 1998;18:229-35.

11. Puddu PE, Brancaccio G, Leacche M, Monti F, Lanti M, et al. Prediction of early and delayed postoperative deaths after coronary artery bypass surgery alone in Italy. Multivariate predictions based on Cox and logistic models and a chart based on the accelerated failure time model. Ital Heart J 2002;3:166-81.

12. Edwards FH, Clark RE, Schwartz M. Coronary artery bypass grafting: the society of thoracic surgeons national database experience. Ann Thorac Surg 1994;57:12-9.

13. O'Brien SM, Feng L, He X, Xian Y, Jacobs JP, et al. The society of thoracic surgeons 2018 adult cardiac surgery risk models: part 2-statistical methods and results. Ann Thorac Surg 2018;105:1419-28.

14. Shahian DM, Jacobs JP, Badhwar V, Kurlansky PA, Furnary AP, et al. The society of thoracic surgeons 2018 adult cardiac surgery risk models: part 1-background, design considerations, and model development. Ann Thorac Surg 2018;105:1411-8.

15. Wu C, Camacho FT, Wechsler AS, Lahey S, Culliford AT, et al. Risk score for predicting long-term mortality after coronary artery bypass graft surgery. Circulation 2012;125:2423-30.

16. Head SJ, da Costa BR, Beumer B, Stefanini GG, Alfonso F, et al. Adverse events while awaiting myocardial revascularization: a systematic review and meta-analysis. Eur J Cardiothorac Surg 2017;52:206-17.

17. Collins D, Goldberg S. Care of the post-CABG patient. Cardiol Rev 2020;28:26-35.

18. Engel J, Damen NL, van der Wulp I, de Bruijne MC, Wagner C. Adherence to cardiac practice guidelines in the management of non-STelevation acute coronary syndromes: a systematic literature review. Curr Cardiol Rev 2017;13:3-27.

19. Pinho-Gomes AC, Azevedo L, Ahn JM, Park SJ, Hamza TH, et al. Compliance with guideline-directed medical therapy in contemporary coronary revascularization trials. J Am Coll Cardiol 2018;71:591-602.

20. Salari A, Hasandokht T, Mahdavi-Roshan M, Kheirkhah J, Gholipour M, et al. Risk factor control, adherence to medication and follow up visit, five years after coronary artery bypass graft surgery. J Cardiovasc Thorac Res 2016;8:152-7.

21. Blumenthal JA, Lett HS, Babyak MA, White W, Smith PK, et al. Depression as a risk factor for mortality after coronary artery bypass surgery. Lancet 2003;362:604-9.

22. Connerney I, Sloan RP, Shapiro PA, Bagiella E, Seckman C. Depression is associated with increased mortality 10 years after coronary artery bypass surgery. Psychosom Med 2010;72:874-81.

23. Charles EJ, Mehaffey JH, Hawkins RB, Fonner CE, Yarboro LT, et al.; Investigators for the Virginia Cardiac Services Quality I. Socioeconomic distressed communities index predicts risk-adjusted mortality after cardiac surgery. Ann Thorac Surg 2019;107:1706-12. 